\title{
From the N-body Schrödinger equation to the quantum Boltzmann equation: a term-by-term convergence result in the weak coupling regime.
}

\author{
D. BENEDETTO ${ }^{(1)}$, F. CASTELLA ${ }^{(2)}$, \\ R. ESPOSITO ${ }^{(3)}$, M. PULVIRENTI ${ }^{(1)}$ \\ (1) Dipartimento di Matematica - Università di Roma 'La Sapienza' \\ P.le Aldo Moro, 5 - 00185 ROMA - ITALY \\ (2) IRISA \& IRMAR - Université de Rennes 1 \\ Campus de Beaulieu - 35042 RENNES - CEDEX - FRANCE \\ (3) Dipartimento di Matematica pura ed applicata - Università di L'Aquila \\ Coppito - 67100 L'AQUILA - ITALY
}

Comm. Math. Phys., Vol. 277, N. 1, pp. 1-44 (2008).

\begin{abstract}
In this paper we analyze the asymptotic dynamics of a system of $N$ quantum particles, in a weak coupling regime. Particles are assumed statistically independent at the initial time.

Our approach follows the strategy introduced by the authors in a previous work [BCEP1]: we compute the time evolution of the Wigner transform of the one-particle reduced density matrix; it is represented by means of a perturbation series, whose expansion is obtained upon iterating the Duhamel formula; this approach allows us to follow the arguments developed by Lanford [L] for classical interacting particles evolving in a low density regime.

We prove, under suitable assumptions on the interaction potential, that the complete perturbation series converges term-by-term, for all times, towards the solution of a Boltzmann equation.

The present paper completes the previous work [BCEP1]: it is proved there that a subseries of the complete perturbation expansion converges uniformly, for short times, towards the solution to the nonlinear quantum Boltzmann equation. This previous result holds for (smooth) potentials having possibly non-zero mean value. The present text establishes that the terms neglected at once in [BCEP1], on a purely heuristic basis, indeed go term-by-term to zero along the weak coupling limit, at least for potentials having zero mean.

Our analysis combines stationary phase arguments, with considerations on the nature of the various Feynman graphs entering the expansion.
\end{abstract}

\section{Introduction}

As it is well known, a large particle system in a rarefaction regime should be described by a Boltzmann equation, be it in the context of quantum or classical mechanics. However, while the rigorous validity of the Boltzmann equation has been proved for classical systems for short times [L], or globally in time for special situations [IP] (see Ref. [CIP] for further comments), there is no fully rigorous analysis for the equivalent quantum systems. The problem is physically relevant yet, because quantum effects, although usually negligible at ordinary temperatures (except for few light molecules), happen to play a role in the applications at mesoscopic level. We refer, for example, to the treatment of electron gases in semiconductors (for physical references, see the textbooks $[\mathrm{RV}],[\mathrm{AM}],[\mathrm{Ch}]$, as well as [Bo], [CTDL] - see [MRS] for a mathematically oriented presentation). 
Therefore, establishing a well founded quantum kinetic theory is certainly interesting not only from a conceptual viewpoint but also from a practical one. In fact, kinetic descriptions for quantum systems, beside dilute gases, include dense weakly interacting systems, as e.g. electron gas in semiconductors, whose classical analogues rather yield diffusion processes in velocity, described by the Landau equation.

One pragmatic way to introduce the quantum Boltzmann equation (see e.g. [CC]) is to solve the scattering problem in quantum mechanics and then to replace, in the classical Boltzmann equation, the classical cross section with the quantum one.

A better logically founded approach is to consider the kinetic like equation for the Wigner transform of a quantum state associated to a particle system. Such a transform, defined on the classical phase space, should converge towards the solution of a nonlinear quantum Boltzmann equation along the natural asymptotic process. The mathematical challenge is to prove such a convergence result.

This is the strategy we adopted in the previous papers [BCEP1], [BCEP2], [BCEP3].

In [BCEP1], we consider a quantum $N$ particles system in the weak coupling regime (see below for the definition). The key point is, we prove the Wigner transforms of the reduced density matrices satisfy a hierarchy of equations that is similar to the so-called BBGKY hierarchy encountered in the context of classical interacting particles. Besides we prove that the arguments developed by Lanford [L] in the classical context may be partly reproduced: the hierarchy may be solved iteratively, and we may write an explicit expression for the $j$-particles reduced Wigner transforms at any time $t$, as a function of the initial state of the system; this expression involves a huge series expansion which is naturally indexed by graphs; the latter are of the Feynman graph type; they take into account all possible particles' interactions between time 0 and time $t$.

This basic observation is the common ingredient in [BCEP1], [BCEP2], [BCEP3], and the present text.

Now, the situation is as follows. In the classical context, Lanford [L] has shown it is possible to pass to the limit directly in the so obtained series expansion, and the convergence is uniform for short time. We refer to the book [CIP] for additional remarks. One important feature naturally is that the trajectories of classical particles are quite explicitly known in the classical context. In the quantum context particles become delocalized and their various interactions are much more delicate to enumerate. In particular, the delocalization effect gives rise to various highly oscillatory phase factors which have to be analyzed. Due to these new analytical difficulties, the result we proved in [BCEP1] is not a complete convergence result: we prove a subseries (of the complete series expansion expressing the state of the system at time $t$ ) converges, along the weak coupling limit, towards the solution of the desired quantum Boltzmann equation. The convergence is uniform for short times. We also present in [BCEP1] arguments of heuristic nature which tend to establish the terms we neglect at once, when passing from the complete series expansion to the retained subseries, indeed go to zero along the weak coupling limit.

Starting from the same observation, the analysis is extended in [BCEP3] to tackle the more delicate low density regime (see below for the definition). There, the new difficulty lies in the identification of the so-called cross-section entering the limiting Boltzmann equation, and we refer to this text for the details. The result in [BCEP3] again is a partial result: a subseries of the complete series expansion (expressing the solution at time $t$ ) is proved to converge, uniformly for short times, towards the natural limit.

Last, while the works [BCEP1] and [BCEP3] tackle the case of statistically independent particles, the analysis is also extended in [BCEP2] to handle the physically realistic case of bosons or fermions: such particles are not statistically independent (they obey the Bose-Einstein, resp. Fermi-Dirac statistics), and the limiting Boltzmann equation needs to be modified accordingly [UU] (see also [HL], [ESY], $[\mathrm{H}]$ )). Again, a partial convergence result towards the conveniently corrected quantum Boltzmann equation is established in [BCEP2] along the similar lines.

Note that the situation is much better understood in the linear context, namely that of a single particle in a given random field: here the limiting equation is a linear Boltzmann equation, which has been rigorously derived for short times ([Sp]) and, more recently, globally in time (see [EY1, EY2], see also [EE] for the low density regime).

With this overall picture in mind, the main contribution of the present text is the following: as in [BCEP1], we consider the weak coupling limit for statistically independent particles; we first write down the complete series expansion, or more precisely the Feynman graph expansion, which relates the state of the system at time $t$ 
as a function of its initial value; we analyze the term-by-term limit of the full expansion; we eventually establish the limiting value satisfies the correct quantum Boltzmann equation. In other words, we are able to prove here that the terms we neglected at once in [BCEP1], when passing from the full expansion to a subseries, indeed go to zero term-by-term along the weak coupling limit.

Two important new points allow us the present extension of the analysis performed in [BCEP1]. On the one hand, we are able to put forward a general stationary phase argument that allows to treat in full generality the highly oscillatory phase factors entering the complete series expansion. On the other hand, we are able to characterize the various Feynman graphs entering in the expansion.

Unfortunately, our analysis still has two weaknesses, yet.

First, the present result states a term-by-term convergence only, and uniform bounds are still missing. This is mainly due to the following fact: the graphs that naturally index the complete series expansion are "too numerous" at each order of the expansion; this gives rise to "too large" combinatorial factors in the analysis; in turn, this phenomenon prevents us from being able to provide a clear uniform bound. In any circumstance, we remark that the term-by-term convergence is a conceptually delicate point: the irreversible nature of the Boltzmann equation compared with the time-reversible character of the Schrödinger evolution emerges exactly there. This aspect of the analysis is clear in the Lanford proof, performed for classical particles in the low density regime. In that case, the term-by-term convergence follows easily by "direct inspection", due to the fact that the classical evolution is somehow explicit. At variance, and as pointed out by Uchiyama (see [U], and [CIP] for further comments), the natural Hamiltonian dynamics formally leading to the two dimensional Broadwell model, a very similar model with similar convergence issues, fails to converge at the fourth order term of the perturbation expansion, showing the validation of the Broadwell equation to be false.

The second point is, our result requires the elementary interaction potential has zero mean value. This condition appears naturally in the analysis. It prevents a singularity associated with "collisions" corresponding to an exchange of zero momentum. This singularity is probably the analog of the grazing collision singularity which occurs in the classical case. However, while it is a standard fact that one can remove this singularity in the classical framework, upon assuming the potential is short range, in the quantum framework the short range assumption is not enough because of the particles' delocalization: the latter makes the effective interaction range actually infinite, even for compactly supported potentials. Now, the observed singularity that comes up in our analysis, which is associated with particles exchanging zero momentum, should in principle be removed by compensation between the gain and loss terms. Yet making this statement rigorous requires regularity conditions which are hard to propagate along a general graph. For this reason, we give up with such a strategy and make the restrictive hypothesis of a potential having zero average. This is certainly not optimal, but it has the advantage of providing a sufficiently simple proof.

Finally, we point out that the results we present here are limited to the unphysical case of particles obeying the Maxwell-Boltzmann statistics. In other words our analysis, based on computations that are fully expressed in classical phase space, requires particles be described by fully factorized initial states of the form (see (2.17) below)

$$
f_{j}^{N}\left(t=0, x_{1}, v_{1}, \ldots, x_{j}, v_{j}\right) \equiv \prod_{k=1}^{j} f_{0}\left(x_{j}, v_{j}\right),
$$

where we refer to section 2 for the precise definitions and notation, and simply mention $f_{j}^{N}(0)$ roughly is the $j$-particles distribution function of the underlying $j$-particles system at the initial time, while $f_{0}$ is a given one-particle density function.

We wish to discuss here how our approach may be extended to the physical situation of particles obeying either the Bose-Einstein or the Fermi-Dirac statistics.

As shown in [BCEP2], taking into account the statistical dependence between particles requires to modify in a deep way the structure of the initial state. Indeed, product states of the form (1.1) are not relevant for fermionic nor bosonic particles, because the associated statistics creates correlations. More precisely, free Bosons 
or Fermions are usually described by states which are usually called "quasi-free" and involve, in some sense, the minimal correlations that are compatible with the quantum statistics. The remarkable fact is, one may fully characterize these states in classical phase space: the $j$-particles distribution function of a quasi-free state is of the form

$$
f_{j}^{N}(0) \equiv \sum_{\pi}( \pm 1)^{s(\pi)}\left(f_{j}^{N}(0)\right)^{\pi}
$$

where $\pi$ is the generic permutation between indices $\{1,2, \ldots, j\}, s(\pi)$ is its signature, $\pm 1 \equiv 1$ for bosons, and $\pm 1 \equiv-1$ for fermions, while we have set

$$
\begin{aligned}
\left(f_{j}^{N}(0)\right)^{\pi}\left(x_{1}, v_{1}, \ldots, x_{j}, v_{j}\right) \equiv \frac{1}{(2 \pi)^{3 j}} \int d y_{1} \ldots \int d y_{j} \int d w_{1} \ldots \int d w_{j} \\
\quad \prod_{k=1}^{j}\left(\mathrm{e}^{i y_{k} \cdot v_{k}+i w_{k} \cdot \frac{x_{k}-x_{\pi(k)}}{\varepsilon}-i w_{k} \cdot \frac{y_{k}+y_{\pi(k)}}{2}} f_{0}\left(\frac{x_{k}+x_{\pi(k)}}{2}-\frac{\varepsilon}{4}\left(y_{k}-y_{\pi(k)}\right), w_{k}\right)\right) .
\end{aligned}
$$

Here, $\varepsilon$ is the small parameter entering the weak-coupling limit we discuss throughout this text (see section 2). Note that expression (1.3) is formula (2.29) in [BCEP2]. A simple stationary phase analysis shows each

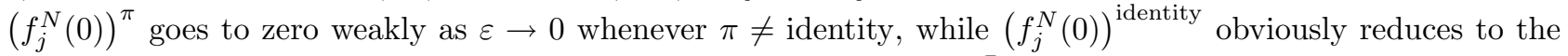
tensor product in (1.1). In other words, the correlation terms $\left(f_{j}^{N}(0)\right)^{\pi}$ with $\pi \neq$ identity are definitely small in a weak topology due to the fact they involve fast oscillating phase factors. Yet at later times $t>0$, correlations do induce finite macroscopic effects caused by the interaction between particles: the expected limiting kinetic equation in the case of Fermions/Bosons is the Uehling-Uhlembeck equation, which corrects the structure of the usual collision operator, a quadratic term in the distribution function, by cubic contributions. We have actually proved in [BCEP2] that the quantum dynamics indeed agrees, in the weak-coupling limit, with the Uehling-Uhlembeck equation up to the second order terms (in the potential) of the perturbative expansion.

In this picture, the generalization of the present paper's result to Bosons and Fermions is highly nontrivial. Indeed, our basic approach relies on a complete Feynman graph expansion of the solution at time $t$, where each term of the expansion turns out to be associated with a specific highly oscillatory phase factor, the latter being analyzed using algebraic and stationary phase analytic arguments. The set of graphs (and associated phase factors) to be considered is obviously much larger in the case of Fermions/Bosons, due to the contribution of all permutations $\pi$ in (1.2)-(1.3): the present paper only estimates those terms stemming from the permutation $\pi=$ identity. However, the analysis in [BCEP1], [BCEP2], and that in the present paper, allow us to conjecture what is the family of graphs which gives the correct equation, and how one may show the other terms are vanishing. In this direction yet, there is still another difficulty that is specific to the case of fermionic/bosonic particles: as observed in [BCEP2], some of the new graphs associated with correlation terms are actually diverging as $\varepsilon \rightarrow 0$, and only subtle cancellation effects between such terms allow to prove the latter still do not contribute to the limit. In conclusion, although the case of bosonic/fermionic particles can certainly be approached with the present techniques, it is still open at this stage, and its solution requires a technical effort supported by new algebraic and analytical ideas.

The paper is organized as follows.

In section 2, we first present the basic model, the associated scaled Schrödinger equation, and its Wigner transform. The latter solves a $N$-particles kinetic like equation. We next derive the quantum BBGKY hierarchy that is associated with this kinetic model. We solve it iteratively as discussed before, and express its solution as a complete series expansion. This part of the discussion gathers arguments previously developed in [BCEP1]. On the other hand, we also present the limiting quantum Boltzmann equation that is to be derived, and express its solution as a complete series expansion as well, using an iterative argument. At this level, the solution of the interacting particles system, and the solution of the Boltzmann equation, are given through two different series expansions. With these expressions at hand, we state our main term-by-term convergence result.

The remainder part of the paper is dedicated to the proof of our main result.

Section 3 is mainly devoted to presenting the Feynman graph interpretation of the various series expansions, and section 4 is devoted to sorting out the graphs. Each Feynman graph represents a different interaction history, between time $t$ and the initial time. 
Section 5 presents three Lemmas which allow for a very precise description of the various impulse exchanges along the particles' interactions, depending on the type of Feynman graph (or: collision history) we are dealing with.

Last, sections 6 through 9 are devoted to the analysis of four different types of graphs, corresponding to a natural partition of all possible graphs. The first two types (sections 6 and 7) are treated using a general stationary phase argument, in conjunction with the combinatorial information discussed in the previous section 5. The last two types (sections 8 and 9) are treated using the fact the potential has zero mean value: it allows to balance a natural singularity in the problem, and to conclude at once. Again, this part of the analysis uses the combinatorial information discussed in section 5 .

The present study holds in any dimension $d \geq 3$.

\section{The model and its scaling limit - Statement of our main result}

\subsection{The $N$-body Schrödinger equation in the weak coupling regime}

We consider a quantum system of $N$ identical particles in $\mathbb{R}^{d}$, located at the positions $x_{i} \in \mathbb{R}^{d}(i=1, \ldots, N)$. We assume the mass of the particles, as well as $\hbar$, are normalized to unity. We also assume all pairs of particles interact through the same two body potential $\phi$. The potential energy of the full $N$-particles system is

$$
U\left(x_{1}, \ldots, x_{N}\right)=\sum_{i<j} \phi\left(x_{i}-x_{j}\right) .
$$

The $N$-body Schrödinger equation associated with the potential $U$ reads

$$
i \partial_{t} \Psi\left(t, X_{N}\right)=-\frac{1}{2} \Delta_{N} \Psi\left(t, X_{N}\right)+U\left(X_{N}\right) \Psi\left(t, X_{N}\right)
$$

where $\Delta_{N}=\sum_{i=1}^{N} \Delta_{x_{i}}$ and $\Delta_{x_{i}}$ is the Laplacian with respect to variable $x_{i}$, while $X_{N} \in \mathbb{R}^{d N}$ is a shorthand notation for the collection of positions $X_{N}=\left(x_{1}, \ldots, x_{N}\right)$. Equation $(2.2)$, when supplemented with the appropriate initial datum $\Psi\left(0, X_{N}\right)$, completely determines the dynamics of the quantum $N$-particles system under consideration.

We are interested in the asymptotic behavior of the system (2.2) in the weak coupling regime, which we now describe.

On the one hand, we rescale the Schrödinger equation (2.2) according to the hyperbolic space-time scaling

$$
x \mapsto \varepsilon x, \quad t \mapsto \varepsilon t,
$$

where $\varepsilon$ is a small dimensionless parameter. Simultaneously, we also rescale the potential as

$$
\phi \mapsto \sqrt{\varepsilon} \phi
$$

Last, we impose the following relation between the large number $N$ of particles, and the small parameter $\varepsilon$, namely

$$
N=\varepsilon^{-d} .
$$

The whole rescaling $(2.3),(2.4),(2.5)$ means the density of particles, i.e. the typical number of particles per unit volume, is kept fixed of the order of unity. It also means we are interested in the behavior of the $N$-particles system over long times, of the order of $1 / \varepsilon$, in the case when the interaction potential $\phi$ is weak, of the order of $\sqrt{\varepsilon}$. Incidentally, our time rescaling forces the associated space rescaling: particles travel at a speed of the order of unity, and we need to look at the behavior of the system over long distances of the order $1 / \varepsilon$ as well. 
The resulting scaled form of equation $(2.2)$ is

$$
\begin{aligned}
& i \varepsilon \partial_{t} \Psi^{\varepsilon}\left(t, X_{N}\right)=-\frac{\varepsilon^{2}}{2} \Delta_{N} \Psi^{\varepsilon}\left(t, X_{N}\right)+U_{\varepsilon}\left(X_{N}\right) \Psi^{\varepsilon}\left(t, X_{N}\right), \\
& \text { where } U_{\varepsilon}\left(x_{1}, \ldots, x_{N}\right)=\sum_{i<j} \phi_{\varepsilon}\left(x_{i}-x_{j}\right), \quad \phi_{\varepsilon}=\sqrt{\varepsilon} \phi\left(\frac{x}{\varepsilon}\right), \quad N=\varepsilon^{-d} .
\end{aligned}
$$

Again, $\Psi^{\varepsilon}\left(t, X_{N}\right)$ is fully determined by Eq. (2.6) and the initial datum $\Psi^{\varepsilon}\left(0, X_{N}\right)$, which is specified later on (see (2.17) below).

The aim of this text is to perform the limit $\varepsilon \rightarrow 0$ in (2.6)-(2.7), and to identify the asymptotic dynamics. We wish to prove the above system tends to be well described by a nonlinear Boltzmann equation (2.29) in the limit $\varepsilon \rightarrow 0$, as physically expected in view of the comments below.

The present limit is usually called a weak-coupling limit. It is characterized by the fact the potential interaction is weak, of order $\sqrt{\varepsilon}$, and the density of particles is 1 . Therefore the number of collision per unit time is $\varepsilon^{-1}$. Since the quantum mechanical cross-section in the Born approximation (justified because the potential is small) is quadratic in the potential interaction, the cumulated effect is of the order

$$
\text { number of collisions } \times[\text { potential interaction }]^{2}=1 / \varepsilon \times \varepsilon=1 \text {. }
$$

We also comment on the weak coupling regime for classical systems. Here a test particle suffers $\varepsilon^{-1}$ small collision per unit time. Now the limiting equations is expected to be the so called Fokker-Plank-Landau equation which describes a diffusion in velocity. No rigorous result is known up to now in this direction, although the linear case (a single particle moving under the action of an external random field) is well understood ([KP, DGL]), and exhibits the expected diffusive behavior. The different structure of the one particle kinetic equation for classical (diffusion in velocity) and quantum (jumps in velocity, as given by a Boltzmann equation) is consequence of the different nature of the scattering mechanism for a single classical and quantum particle. Indeed a quantum particle has a small probability to be deflected by any angle for each collision, and the cumulative effect is a jump. On the contrary, a classical particle is always deflected by a small angle, and the cumulative effect yields a diffusion.

Another possible scaling to be considered is the low-density limit. In this case $\phi=O(1)$ is unscaled but $N=O\left(\varepsilon^{-d+1}\right)$. This again results in a cumulated effect of the order of unity, yet the picture is different: particles "collide" only once per unit time in this scaling, but each "collision" now has a dominant effect, of the order unity at once. In the classical context this is nothing but the Boltzmann-Grad limit (see e.g. [CIP]). We also refer to $[\mathrm{C}]$ for the analysis of a low-density situation in a linear context.

The present paper is only concerned with the weak coupling limit. As mentioned in the introduction, we prove here a term-by-term convergence result. It completes the partial convergence result previously obtained in [BCEP1]. On the other hand, we prove in [BCEP3] a partial convergence result concerning the low density regime. Due to the fact the potential interactions somehow are weaker in the weak coupling regime than in the low density regime, the former case only involves $\phi$ at lower order (the cross-section obtained in the eventual Boltzmann equation is proportional with $\phi^{2}$, see below), while the latter requires to consider a full series expansion in $\phi$ (the so-called Born series expansion). It in turn needs to be identified and summed up in the appropriate way, and the difficulties linked with the necessary control and identification of the Born series expansion are treated in [BCEP3]. We do not know whether the techniques we develop in the present text may be adapted to transform the partial convergence result of [BCEP3] into a complete term-by-term convergence result, valid in the low density regime as well.

\subsection{Transforming the $N$-body Schrödinger equation into a hierarchy of kinetic equations}

In order to tackle the asymptotic analysis of the scaled Schrödinger (2.6)-(2.7), we follow the approach we introduced in [BCEP1]. 
We consider the Wigner function $f^{N}\left(t, X_{N}, V_{N}\right)$ associated with the wave function $\Psi^{\varepsilon}\left(t, X_{N}\right)$, solution to the $N$-body Schrödinger equation (2.6)-(2.7). It is defined, see [W], as

$$
f^{N}\left(t, X_{N}, V_{N}\right)=\left(\frac{1}{2 \pi}\right)^{d N} \int_{\mathbb{R}^{d N}} d Y_{N} \mathrm{e}^{i Y_{N} \cdot V_{N}} \bar{\Psi}^{\varepsilon}\left(t, X_{N}+\frac{\varepsilon}{2} Y_{N}\right) \Psi^{\varepsilon}\left(t, X_{N}-\frac{\varepsilon}{2} Y_{N}\right),
$$

and $V_{N} \in \mathbb{R}^{d N}$ is a shorthand notation for the velocity variable $V_{N}=\left(v_{1}, \ldots, v_{N}\right)$. The quantity $f^{N}$ roughly is the distribution function, in phase-space, of the $N$-particles system under study, although a delicate point is $f^{N}$ does not have a definite sign yet. We refer e.g. to [Ba] for the general properties of the Wigner function and $[\mathrm{LP}]$ for more sophisticated considerations on the mathematical side.

A standard computation establishes that $f_{N}=f_{N}\left(t, X_{N}, V_{N}\right)$ satisfies a kinetic transport equation, namely

$$
\left(\partial_{t}+\sum_{k=1}^{N} v_{k} \cdot \nabla_{x_{k}}\right) f^{N}=\frac{1}{\sqrt{\varepsilon}} T_{N}^{\varepsilon} f^{N}
$$

where the operator $\partial_{t}+\sum_{k=1}^{N} v_{k} \cdot \nabla_{x_{k}}$ is the usual free stream operator, and we have introduced the crucial operator

$$
\begin{aligned}
& T_{N}^{\varepsilon}=\sum_{1 \leq r<\ell \leq N} T_{r, \ell}^{\varepsilon}, \quad \text { with } \\
& \left(T_{r, \ell}^{\varepsilon} f^{N}\right)\left(t, X_{N}, V_{N}\right)=-i \sum_{\sigma= \pm 1} \sigma \int_{\mathbb{R}^{d}} \frac{d h}{(2 \pi)^{d}} \mathrm{e}^{i h \cdot\left(x_{r}-x_{\ell}\right) / \varepsilon} \widehat{\phi}(h) \\
& f^{N}\left(t, X_{N}, v_{1}, \ldots, v_{r-1}, v_{r}-\sigma \frac{h}{2}, v_{r+1}, \ldots, v_{\ell-1}, v_{\ell}+\sigma \frac{h}{2}, v_{\ell}, \ldots, v_{N}\right) .
\end{aligned}
$$

The reader may note the scaled Schrödinger equation (2.6)-(2.7) is not a semi-classical equation: the interaction potential is $\phi(. / \varepsilon)$ instead of $\phi$. This explains the fact that equation (2.9) is not similar to the transport equation along the Hamiltonian flow associated with $\phi$ : the interaction is here varying on the quantum scale, not on the semi-classical one.

Equation (2.9) actually asserts the dynamics of $f^{N}$ is governed by two effects: free transport on the one hand (this is the left hand side of (2.9)), and internal "collisions" inside the particles' system on the other hand (this is the right hand side of (2.9)). The operator $T_{r, \ell}^{\varepsilon}$ describes the "collision" of particle $r$ with particle $\ell$, and the total operator $T_{N}^{\varepsilon}$ takes all possible "collisions" into account. "Collisions" involve a momentum transfer $h$. They may occur at distant places $\left(x_{r} \neq x_{\ell}\right)$, which typically is a quantum feature. This fact is balanced by the highly oscillatory factor $\exp \left(i h \cdot\left(x_{r}-x_{\ell}\right) / \varepsilon\right)$, a natural counterpart.

Here and below, $\widehat{f}$ denotes the Fourier transform of $f$, normalized as follows

$$
\widehat{f}(h)=\left(\mathcal{F}_{x} f\right)(h)=\int_{\mathbb{R}^{d}} d x \mathrm{e}^{-i h \cdot x} f(x), \quad f(x)=\int_{\mathbb{R}^{d}} \frac{d h}{(2 \pi)^{d}} \mathrm{e}^{i h \cdot x} \widehat{f}(h) .
$$

Still following the approach introduced in [BCEP1], we next introduce the partial traces of the Wigner transform, according to the formula

$$
\begin{aligned}
f_{j}^{N}\left(t, X_{j}, V_{j}\right)= & \int_{\mathbb{R}^{d(N-j)}} d x_{j+1} \ldots d x_{N} \int_{\mathbb{R}^{d(N-j)}} d v_{j+1} \ldots d v_{N} \\
& f^{N}\left(t, X_{j}, x_{j+1}, \ldots, x_{N} ; V_{j}, v_{j+1}, \ldots, v_{N}\right),
\end{aligned}
$$

whenever $1 \leq j \leq N-1$. We also set the convention $f_{N}^{N} \equiv f^{N}, \quad f_{N+1}^{N} \equiv 0$. In relation with $f^{N}$, the function $f_{j}^{N}$ roughly is the distribution function in phase-space of the $j$-particles subsystem, inside the larger $N$-particles system under study. 
From now on we shall suppose that, due to the fact that particles are identical, the objects we have introduced $\left(\Psi^{\varepsilon}, f_{j}^{N}\right)$ are all symmetric in the exchange of particles. This assumption is satisfied by fully uncorrelated quantum particles, since they obey the Maxwell-Boltzmann statistics. The symmetry actually makes all $j$ particles subsystems equivalent (they do not depend on the very $j$ particles that have been selected). This justifies in passing the notation " $f_{j}^{N}$ " without further reference to the particles' names.

Proceeding then as in the derivation of the BBKGY hierarchy for classical systems (see [CIP]), it is readily deduced from $(2.9)$ that $f_{j}^{N}=f_{j}^{N}\left(t, X_{j}, V_{j}\right)$ satisfies the following hierarchy of equations

$$
\left(\partial_{t}+\sum_{k=1}^{j} v_{k} \cdot \nabla_{k}\right) f_{j}^{N}=\frac{1}{\sqrt{\varepsilon}} T_{j}^{\varepsilon} f_{j}^{N}+\frac{N-j}{\sqrt{\varepsilon}} C_{j+1}^{\varepsilon} f_{j+1}^{N}, \quad(1 \leq j \leq N),
$$

where $T_{j}^{\varepsilon}$ has been defined before (2.10)-(2.11), and the new operator $C_{j+1}^{\varepsilon}$, is

$$
\begin{aligned}
& C_{j+1}^{\varepsilon}=\sum_{k=1}^{j} C_{k, j+1}^{\varepsilon}, \quad \text { with } \\
& \begin{array}{l}
\left(C_{k, j+1}^{\varepsilon} f_{j+1}^{N}\right)\left(t, X_{j}, V_{j}\right)=-i \sum_{\sigma= \pm 1} \sigma \int_{\mathbb{R}^{d}} \frac{d h}{(2 \pi)^{d}} \widehat{\phi}(h) \int_{\mathbb{R}^{d}} d x_{j+1} \int_{\mathbb{R}^{d}} d v_{j+1} \\
\quad \mathrm{e}^{i \frac{h}{\varepsilon}\left(x_{k}-x_{j+1}\right)} f_{j+1}^{N}\left(t, X_{j}, x_{j+1}, v_{1}, \ldots, v_{k-1}, v_{k}-\sigma \frac{h}{2}, v_{k+1}, \ldots, v_{j}, v_{j+1}+\sigma \frac{h}{2}\right) .
\end{array}
\end{aligned}
$$

Note that (2.9) is recovered from (2.14) upon setting $j=N$. The operator $C_{k, j+1}^{\varepsilon}$ describes the "collision" of particle $k$, belonging to the $j$-particle subsystem, with a generic particle outside the subsystem, conventionally denoted by the number $j+1$. This conventional numbering uses the fact all particles are identical. The total operator $C_{j+1}^{\varepsilon}$ takes all such collisions into account. As usual [CIP], equation (2.14) shows the dynamics of the $j$-particle subsystem is governed by three effects: the free-stream operator, the collisions "inside" the subsystem (the $T$ term), and the collisions with particles "outside" the subsystem (the $C$ term).

Last, there remains to determine the initial value $\left\{f_{j}^{N}(0)\right\}_{j=1}^{N}$ of the solution $\left\{f_{j}^{N}(t)\right\}_{j=1}^{N}$. We assume $\left\{f_{j}^{N}(0)\right\}_{j=1}^{N}$ is factorized, that is, for all $j=1, \ldots, N$

$$
f_{j}^{N}(0)=f_{0}^{\otimes j}
$$

where $f_{0}$ is a one-particle Wigner function which we also assume to be a probability distribution.

These assumptions require comments. The factorization property simply means we are assuming, as usual, the particles be fully uncorrelated at the initial time. Moreover we remind a quantum state whose Wigner transform is a probability distribution is not in general a wave function but rather a density matrix. As a consequence the evolution equation we have to start with is not the Schrödinger equation (2.2) but the associated Heisenberg equation for the density matrix. In both cases the corresponding Wigner equation is anyhow equation (2.9), and the analysis remains unchanged.

We are now considering $f_{0}$ as fixed, but we could also assume an initial condition depending on $\varepsilon$, as happens, for instance, if the one particle state is a suitable smooth superposition of coherent states. Our analysis would change in a minor way. Finally we remark that equation (2.17) is only compatible with the Maxwell-Boltzmann statistics, but not with the Bose-Einstein nor Fermi-Dirac statistics, for which the derivation of kinetic equations involves extra difficulties related to quantum correlations. The analysis we present here cannot be adapted in a direct way to the case of fermionic nor bosonic particles. New difficulties arise in these two situations and the limiting Boltzmann equation (2.29) below actually needs to be modified then. Roughly speaking for Bosons and Fermions we need to consider many more relevant Feynman diagrams. We may quote [BCEP2] for a rigorous analysis up to the second order term. We also refer to the introduction on that point. 


\subsection{Solving the hierarchy}

Up to now we have simply transformed the original Schrödinger equation (2.6) into a hierarchy of equations (2.14). Our approach lies in performing the asymptotic process $\varepsilon \rightarrow 0$ on the hierarchy itself, rather than on the original equation (2.6).

Let us first develop some preliminary considerations in order to have an idea of the size of the operators entering the hierarchy $(2.6)$.

Expanding $f_{j}^{N}(t)$ as a perturbation of the free flow $S(t)$ defined as

$$
\left(S(t) f_{j}\right)\left(X_{j}, V_{j}\right)=f_{j}\left(X_{j}-V_{j} t, V_{j}\right)
$$

we find

$$
f_{j}^{N}(t)=S(t) f_{j}^{0}+\frac{N-j}{\sqrt{\varepsilon}} \int_{0}^{t} S\left(t-t_{1}\right) C_{j+1}^{\varepsilon} f_{j+1}^{N}\left(t_{1}\right) d t_{1}+\frac{1}{\sqrt{\varepsilon}} \int_{0}^{t} S\left(t-t_{1}\right) T_{j}^{\varepsilon} f_{j}^{N}\left(t_{1}\right) d t_{1} .
$$

We now try to keep informations on the limit behavior of $f_{j}^{N}(t)$. To do so, we assume for the moment that the time evolved $j$-particles distributions $f_{j}^{N}(t)$ are smooth, in the sense that the derivatives are uniformly bounded in $\varepsilon$.

First, setting $r=\frac{x_{k}-x_{j+1}}{\varepsilon}$ in eq. (2.16), we recover

$$
\begin{aligned}
C_{j+1}^{\varepsilon} f_{j+1}^{N}\left(X_{j} ; V_{j} ; t_{1}\right)= & -i \varepsilon^{d} \sum_{k=1}^{j} \sum_{\sigma= \pm 1} \sigma \int \frac{d h}{(2 \pi)^{d}} \widehat{\phi}(h) \int d r \int d v_{j+1} e^{i h \cdot r} \\
& \quad f_{j+1}^{N}\left(X_{j}, x_{k}-\varepsilon r ; v_{1}, \ldots v_{k}-\sigma \frac{h}{2}, \ldots v_{j+1}+\sigma \frac{h}{2}\right) \\
= & O\left(\varepsilon^{d+1}\right),
\end{aligned}
$$

provided $D_{v}^{2} f_{j+1}^{N}$ is uniformly bounded. Indeed, setting $\varepsilon=0$ in the integrand, the integration over $r$ produces a Dirac mass $\delta(h)$, hence the integrand is independent of $\sigma$ and the sum over $\sigma$ vanishes. Observing next

$$
\frac{N-j}{\sqrt{\varepsilon}}=O\left(\varepsilon^{-d+\frac{1}{2}}\right),
$$

the second term in the right hand side of (2.19) is hence seen to give a vanishing contribution in the limit $\varepsilon \rightarrow 0$.

Second it also is possible to prove

$$
\frac{1}{\sqrt{\varepsilon}} \int_{0}^{t} S\left(t-t_{1}\right) T_{j}^{\varepsilon} f_{j}^{N}\left(t_{1}\right) d t_{1}
$$

is weakly vanishing (see [BCEP1] for a proof), upon using a stationary phase argument, at least provided $f_{j}^{N}$ is smooth.

We are now facing the following alternative: either the limit is trivial, or the time evolved distributions $f_{j}^{N}(t)$ are not smooth. As a consequence, and since we believe the limit is not trivial (actually we expect to get the Boltzmann equation, according to the previous discussion), a rigorous convergence proof seems problematic. The difficulty in obtaining a-priori estimates on the regularity of the $f_{j}^{N}$ 's induces us to exploit the full series expansion of the solution, in order to rather make use of the regularity of the initial datum itself: it allows to keep advantage of the oscillating phases, and to control the possibly diverging powers of $\varepsilon$.

Let us come to the technical details. As in the case of the Boltzmann-Grad limit for classical systems, we first express the solution $f_{j}^{N}(t)$ to the hierarchy (2.14) as a complete series expansion obtained upon iterating the Duhamel formula, namely

$$
\begin{gathered}
f_{j}^{N}(t)=\sum_{n=0}^{N-j} \frac{(N-j) \ldots(N-j-n+1)}{\varepsilon^{n / 2}} \int_{0}^{t} d t_{1} \ldots \int_{0}^{t_{n-1}} d t_{n} \\
S_{i n t}^{\varepsilon}\left(t-t_{1}\right) C_{j+1}^{\varepsilon} S_{i n t}^{\varepsilon}\left(t_{1}-t_{2}\right) C_{j+2}^{\varepsilon} \ldots S_{i n t}^{\varepsilon}\left(t_{n-1}-t_{n}\right) C_{j+n}^{\varepsilon} S_{i n t}^{\varepsilon}\left(t_{n}\right) f_{0}^{\otimes(j+n)} .
\end{gathered}
$$


This expresses the state of the $j$-particles subsystem at time $t$ as an explicit function of the initial value $f_{0}$. Here $S_{i n t}^{\varepsilon}(t) g_{j}$ is the $j$-particle interacting flow, namely the solution to the initial value problem

$$
\left(\partial_{t}+\sum_{k=1}^{j} v_{k} \cdot \nabla_{x_{k}}\right) S_{i n t}^{\varepsilon}(t) g_{j}=\frac{1}{\sqrt{\varepsilon}} T_{j}^{\varepsilon} S_{i n t}^{\varepsilon}(t) g_{j}, \quad S_{i n t}^{\varepsilon}(0) g_{j}=g_{j}
$$

Note equation (2.24) is nothing else than equation (2.14), with the $C$ term removed.

We may in turn expand $S_{i n t}^{\varepsilon}(t) g_{j}$ as a perturbation of the free flow $S(t) g_{j}$. In doing so we find

$$
\begin{gathered}
S_{i n t}^{\varepsilon}(t) f_{j}=\sum_{m \geq 0} \frac{1}{\varepsilon^{m / 2}} \int_{0}^{t} d t_{1} \int_{0}^{t_{1}} d t_{2} \ldots \int_{0}^{t_{m-1}} d t_{m} S\left(t-t_{1}\right) T_{j}^{\varepsilon} \\
S\left(t_{1}-t_{2}\right) T_{j}^{\varepsilon} \ldots S\left(t_{m-1}-t_{m}\right) T_{j}^{\varepsilon} S\left(t_{m}\right) f_{j} .
\end{gathered}
$$

We insert (2.25) into (2.23). In order to keep reasonably short formulae, we first rewrite (2.23) and (2.25) as, respectively,

$$
\begin{aligned}
& f_{j}^{N}(t)=\sum_{n=0}^{N-j} \frac{(N-j) \cdots(N-j-n+1)}{\varepsilon^{n / 2}} \int\left[S_{i n t}^{\varepsilon} C_{j+1}^{\varepsilon} S_{i n t}^{\varepsilon} C_{j+2}^{\varepsilon} \cdots S_{i n t}^{\varepsilon} C_{j+n}^{\varepsilon} S_{i n t}^{\varepsilon}\right] f_{0}^{\otimes(j+n)} \\
& S_{i n t}^{\varepsilon}=\sum_{m \geq 0} \frac{1}{\varepsilon^{m / 2}}\left(S T_{j}\right)^{m} S .
\end{aligned}
$$

Here, we make an obvious abuse of notation, namely, the integral $\int$ in $(2.26)$ stands for the integration over the set $0 \leq t_{n} \leq t_{n-1} \leq \cdots \leq t_{1} \leq t$, i.e. for $\int_{0}^{t} d t_{1} \int_{0}^{t_{1}} d t_{2} \cdots$ Now, insertion of (2.23) into (2.25) eventually results in the following formula:

$$
\begin{aligned}
& f_{j}^{N}(t)=\sum_{n=0}^{N-j} \sum_{m_{0} \geq 0} \sum_{m_{1} \geq 0} \cdots \sum_{m_{n} \geq 0} \frac{(N-j) \cdots(N-j-n+1)}{\varepsilon^{\left(n+m_{0}+\cdots+m_{n}\right) / 2}} \\
& \int\left[\left(S T_{j}^{\varepsilon}\right)^{m_{0}} S C_{j+1}^{\varepsilon}\left(S T_{j+1}^{\varepsilon}\right)^{m_{1}} S C_{j+2}^{\varepsilon} \cdots\left(S T_{j+n-1}^{\varepsilon}\right)^{m_{n-1}} S C_{j+n}^{\varepsilon}\left(S T_{j+n}^{\varepsilon}\right)^{m_{n}} S\right] f_{0}^{\otimes(j+n)} .
\end{aligned}
$$

Remark 1. For any given value of $N$ and $j$, the normalizing prefactor in (2.27) satisfies

$$
\frac{(N-j) \cdots(N-j-n+1)}{\varepsilon^{\left(n+m_{0}+\cdots+m_{n}\right) / 2}} \underset{\varepsilon \rightarrow 0}{\sim} \varepsilon^{-d n-\left(n+m_{0}+\cdots+m_{n}\right) / 2},
$$

due to the weak-coupling scaling $N \sim \varepsilon^{-d}$.

\subsection{The limiting Boltzmann equation}

In order to state our main result precisely, we next need to introduce some additional notations, in relation with the limiting Boltzmann equation which is to be derived.

It is physically expected that the distribution function $f_{j}^{N}(t)$ converges towards a tensor product $f(t)^{\otimes j}$ as $\varepsilon \rightarrow 0$. This is the so-called "propagation of chaos": initially uncorrelated particles $f_{j}^{N}(0)=f_{0}^{\otimes j}$ tend to remain uncorrelated for all times as $\varepsilon \rightarrow 0$, although the original dynamics tends to actually create correlations between particles through the interaction potential $\phi$. There remains to describe the behavior of the one particle 
distribution function $f(t) \equiv f(t, x, v)$. In turn, it is physically expected that $f(t)$ satisfies the following nonlinear Boltzmann equation ${ }^{1}$

$$
\begin{aligned}
& \partial_{t} f(t, x, v)+v \cdot \nabla_{x} f=Q(f, f)(t, x, v), \quad f(0, x, v)=f_{0}(x, v), \quad \text { where } \\
& Q(f, f)(t, x, v)=\int_{\mathbb{R}^{3} \times \mathbb{S}^{2}} d v_{1} d \omega B\left(\omega, v-v_{1}\right)\left[f\left(t, x, v^{\prime}\right) f\left(t, x, v_{1}^{\prime}\right)-f(t, x, v) f\left(t, x, v_{1}\right)\right], \\
& \text { and the cross-section is } B(\omega, v)=\frac{1}{8 \pi^{2}}|\omega \cdot v||\widehat{\phi}(\omega(\omega \cdot v))|^{2} .
\end{aligned}
$$

Here we have used the standard notations, namely the so-called impact parameter is $\omega \in \mathbb{S}^{2}$, the incoming or pre-collisional velocities are $v \in \mathbb{R}^{3}$ and $v_{1} \in \mathbb{R}^{3}$, and the outgoing or post-collisional velocities are

$$
v^{\prime}=v-\left[v-v_{1}\right] \cdot \omega \quad \omega, \quad v_{1}^{\prime}=v_{1}+\left[v-v_{1}\right] \cdot \omega \quad \omega .
$$

We stress the cross-section $B$ is the only quantum factor in the otherwise purely classical equations (2.29)-(2.30). It retains the quantum features of the elementary "collision events", in that it depends on the microscopic interaction potential $\phi$ through formula (2.31). This relation is known as the "Fermi Golden Rule".

At this level, we have completely determined the physically expected limit $f(t)^{\otimes j}$ of $f_{j}^{N}(t)$ as $\varepsilon \rightarrow 0$, for any given value of $j$.

Since we aim at passing to the limit in $f_{j}^{N}(t)$ in the form given in (2.27), we last need to express $f(t)^{\otimes j}$ in a form that is close to the above expansion (2.27). Computations similar to those performed below for $f_{j}^{N}$ show that the function

$$
f_{j}\left(t, X_{j}, V_{j}\right) \equiv f(t)^{\otimes j}
$$

satisfies a hierarchy of equations (as does $f_{j}^{N}(t)$ ), known under the name of the "Boltzmann hierarchy" see [CIP]. We do not write down the Boltzmann hierarchy for sake of simplicity. Needless to say, the fact that $f_{j}$ and $f_{j}^{N}$ do satisfy parallel hierarchies actually is the main motivation for the kinetic point of view we adopted at once. Now, the Boltzmann hierarchy is easily solved iteratively, as we did for (2.27). Without giving further details (see [BCEP1]), we simply mention $f_{j}(t)$ is given by the following series expansion

$$
\begin{aligned}
f_{j}\left(t, X_{j}, V_{j}\right)=\sum_{n \geq 0} & \int_{0}^{t} d t_{1} \cdots \int_{0}^{t_{n-1}} d t_{n} S\left(t-t_{1}\right) C_{j+1} \\
& \quad S\left(t_{1}-t_{2}\right) C_{j+2} \cdots S\left(t_{n-1}-t_{n}\right) C_{j+n} S\left(t_{n}\right) f_{0}^{\otimes(j+n)},
\end{aligned}
$$

where the operator $S(t)$ is the free flow defined earlier, and the classical collision operator $C_{j+1}$ that is attached with the Boltzmann equation (2.29)-(2.30) is ${ }^{2}$

$$
\begin{aligned}
& C_{j+1}=\sum_{k=1}^{j} C_{k, j+1}, \quad \text { where } \\
& \begin{array}{l}
\left(C_{k, j+1} f_{j+1}\right)\left(t, X_{j}, V_{j}\right):=\int_{\mathbb{R}^{3}} d v_{j+1} \int_{\mathbb{S}_{2}} d \omega B\left(\omega, v_{k}-v_{j+1}\right) \\
\quad\left[f_{j+1}\left(X_{j}, x_{k}, v_{1}, \ldots, v_{k-1}, v_{k}^{\prime}, v_{k+1}, \ldots, v_{j}, v_{j+1}^{\prime}\right)\right. \\
\left.\quad-f_{j+1}\left(X_{j}, x_{k}, v_{1}, \ldots, v_{k-1}, v_{k}, v_{k+1}, \ldots, v_{j}, v_{j+1}\right)\right] .
\end{array}
\end{aligned}
$$

\footnotetext{
${ }^{1}$ The equation is written here in dimension $d=3$ only, for simplicity. Going to the general dimension $d$ only affects the prefactor $1 /\left(8 \pi^{2}\right) \times|\omega \cdot v|$ in $(2.31)$, while the integral that defines $Q(f, f)$ carries over $\mathbb{R}^{d} \times \mathbb{S}^{d-1}$.

${ }^{2}$ Again, formulae are given in dimension $d=3$ only for simplicity.
} 
The cross-section $B$ has been defined in (2.31), and the pre-collisional velocities are $v_{k}^{\prime}=v_{k}-\left[\begin{array}{ll}v_{k}-v_{j+1}\end{array}\right] \cdot \omega \quad \omega$, $v_{j+1}^{\prime}=v_{j+1}+\left[v_{k}-v_{j+1}\right] \cdot \omega \quad \omega$, as in $(2.32)$.

Eventually, and using the same abuse of notations than in $(2.27)$ as far as time integrals $\int_{0}^{t} d t_{1}$ etc. are concerned, we arrive at the following formula for the $j$-particle distribution functions $f_{j}(t) \equiv f(t)^{\otimes j}$ at time $t$, associated with the solution $f(t)$ of the Boltzmann equation (2.29)-(2.30):

$$
f_{j}(t)=\sum_{n \geq 0} \int S C_{j+1} S C_{j+2} \ldots S C_{j+n} S f_{0}^{\otimes(j+n)}
$$

Note that the convergence of the series expansion (2.37), at least for small values of time $t$, is proved in [BCEP1], following [L]. Moreover, proving that $f_{j}^{N}(t)$ "converges" towards $f_{j}(t)$, now amounts to comparing the associated series expansions $(2.27)$ (for $\left.f_{j}^{N}\right)$ resp. $(2.37)$ (for $f_{j}$ ).

\subsection{Statement of the result}

We are in position to state our main result.

\section{Main Theorem}

Assume the initial state $f_{0}$ is smooth, in the sense that the following norm

$$
\mathcal{N}_{\alpha}\left(f_{0}\right)=\sup _{\xi, \eta}\left|\left(1+\xi^{2}+\eta^{2}\right)^{\frac{\alpha}{2}}\left(1-\Delta_{\xi}-\Delta_{\eta}\right)^{\frac{\alpha}{2}} \widehat{f}_{0}(\xi, \eta)\right|
$$

if finite for some $\alpha>2 d$. Assume the potential $\phi$ is smooth, i.e. the following norm ${ }^{3}$

$$
\mathcal{N}_{\alpha}(\phi)=\sup _{h}\left|\left(1+h^{2}\right)^{\frac{\alpha}{2}}\left(1-\Delta_{h}\right)^{\frac{\alpha}{2}} \widehat{\phi}(h)\right|
$$

if finite for some $\alpha>d$. Last, assume the interaction potential $\phi$ has zero mean value, namely

$$
\widehat{\phi}(0)=0 \text {. }
$$

Then, for any given $j \geq 1$, and for any time $t \geq 0$, the series expansion (2.27) that relates the value of $f_{j}^{N}(t)=$ $f_{j}^{N}\left(t, X_{j}, V_{j}\right)$ converges term-by-term towards the series expansion (2.37) that relates the value of $f_{j}(t)=$ $f_{j}\left(t, X_{j}, V_{j}\right)$. The convergence that is mentioned here refers to the fact the Fourier transform $\widehat{f_{j}^{N}}\left(t, \Xi_{j}, H_{j}\right)$ goes to $\widehat{f}_{j}\left(t, \Xi_{j}, H_{j}\right)$ term-by-term, uniformly in $\Xi_{j}$ and $H_{j}$.

\section{A Feynman graph formulation of the problem}

Before coming to the proof of our main Theorem, we first need to reformulate the expansion (2.27) in more appropriate terms.

On the one hand, the expansion (2.27) is more naturally indexed by graphs. They represent all possible interaction histories amongst the $N$ particles, between the initial time $t=0$ and the final time $t$; this is a Feynman diagram expansion. On the other hand, the asymptotic procedure is more easily performed on the Fourier transform of $f_{j}^{N}$ (see the statement of the main Theorem). This point necessitates to reformulate the collision operators $T^{\varepsilon}$ and $C^{\varepsilon}$ in Fourier variables.

The present section is devoted to setting up these two aspects.

For obvious reasons, we shall always restrict our analysis to the one particle distribution function $f_{1}^{N}(t)=$ $f_{1}^{N}\left(t, x_{1}, v_{1}\right)$. Needless to say, the computations can be easily rephrased to prove the more general convergence of $f_{j}^{N}(t)$ towards $f(t)^{\otimes j}$.

\footnotetext{
${ }^{3}$ We use the same notation $\mathcal{N}_{\alpha}$ for the two norms involved in equations (2.38) and (2.39) though, strictly speaking, they do not act on the same function spaces.
} 


\subsection{Graphical representation}

To begin with, since our goal is to pass to the limit term-by-term in $(2.27)$, we observe all prefactors $(N-j) / \sqrt{\varepsilon}$ etc. involved in (2.27) may safely be replaced by $N / \sqrt{\varepsilon}$ at once (all other parameters are considered fixed). Hence, recalling that the weak coupling regime imposes $N \sim \varepsilon^{-d}$, all these prefactors may be replaced by the simpler and equivalent value $\varepsilon^{-d-1 / 2}$.

This being settled, the generic term of the expansion $(2.27)$, called $\mathcal{T}(t)$ in the sequel, always has the form

$$
\mathcal{T}(t)=\varepsilon^{-d(m-1)-n / 2} \int_{0}^{t} d t_{1} \ldots \int_{0}^{t_{n-1}} d t_{n}\left\{S\left(t-t_{1}\right) O_{1}^{\varepsilon} S\left(t_{1}-t_{2}\right) O_{2}^{\varepsilon} \cdots O_{n}^{\varepsilon} S\left(t_{n}\right)\right\} f_{m}^{0}
$$

where $f_{m}^{0}=f_{0}^{\otimes m}$ is the initial datum and each $O_{k}^{\varepsilon}$ is either a $C^{\varepsilon}$ or a $T^{\varepsilon}$ operator. We refer to (2.10)-(2.11) resp. (2.15)-(2.16) for the very definition of $T^{\varepsilon}$ resp. $C^{\varepsilon}$.

We note that, written in that way, the term $\mathcal{T}(t)$ involves $m-1$ operators of type $C^{\varepsilon}$. This freezes the value $m$. It also involves $n-m+1$ operators of type $T^{\varepsilon}$. This freezes the value $n$ which is also the total total number of "collisions". The "collision times" are $t_{1}, t_{2}, \ldots, t_{n}$.

Now, recall the two operators $T^{\varepsilon}$ and $C^{\varepsilon}$ admit the expansions

$$
T_{k}^{\varepsilon}=\sum_{1 \leq r<\ell \leq N}^{k} T_{r, \ell}^{\varepsilon}, \quad C_{k+1}^{\varepsilon}=\sum_{r=1}^{k} C_{r, k+1}^{\varepsilon},
$$

corresponding to all possible particle names $r$ and $\ell$ that are involved in the interactions of each type. As a consequence, keeping the letter $O^{\varepsilon}$ for the generic $C^{\varepsilon}$ or $T^{\varepsilon}$ operator, each $O_{k}^{\varepsilon}$ in (3.1) may be split into

$$
O_{k}^{\varepsilon}=\sum_{r_{k}<\ell_{k}} O_{r_{k}, \ell_{k}}^{\varepsilon}
$$

where $O_{r_{k}, \ell_{k}}^{\varepsilon}$ denotes either $T_{r_{k}, \ell_{k}}^{\varepsilon}$ or $C_{r_{k}, \ell_{k}}^{\varepsilon}$. For that reason, the whole string $\left\{S O_{1}^{\varepsilon} \cdots S O_{n}^{\varepsilon} S\right\}$ in $(3.1)$ may in turn be split into a similar sum. Since the operators $C_{i, k}^{\varepsilon}$ with $k>i$ "creates" the particle $k$, it will be called "creation operator" in the sequel. On the other hand, $T_{i, k}^{\varepsilon}$ will be called "recollision operator" since the particles $i$ and $k$ have already delivered an interaction.

With the above notations, the study of the term-by-term asymptotic behavior of the series expansion (2.27) reduces to that of the generic term

$$
\begin{aligned}
& \mathcal{T}\left(t, x_{1}, v_{1}\right)= \\
& \varepsilon^{-d(m-1)-n / 2} \int_{0}^{t} d t_{1} \ldots \int_{0}^{t_{n-1}} d t_{n}\left\{S\left(t-t_{1}\right) O_{r_{1}, \ell_{1}}^{\varepsilon} S\left(t_{1}-t_{2}\right) O_{r_{2}, \ell_{2}}^{\varepsilon} \cdots O_{r_{n}, \ell_{n}}^{\varepsilon} S\left(t_{n}\right)\right\} f_{m}^{0},
\end{aligned}
$$

for any value of the parameters $m, n$, and any sequence of indices $\left\{\left(r_{k}, \ell_{k}\right)\right\}_{k=1}^{n}\left(r_{k}<\ell_{k}\right)$. Here, each operator $O_{r_{k}, \ell_{k}}^{\varepsilon}$ denotes either $T_{r_{k}, \ell_{k}}^{\varepsilon}$ or $C_{r_{k}, \ell_{k}}^{\varepsilon}$.

The generic term $\mathcal{T}(t)$ is completely determined by the sequence of indices $\left\{\left(r_{k}, \ell_{k}\right)\right\}_{k=1}^{n}$ that is involved in formula (3.4). In this notation the pair $\left(r_{k}, \ell_{k}\right)$ denotes the indices of the particles that actually "collide" at the collision time $t_{k}$, be it a "creation" (operator of type $C^{\varepsilon}$ ) or a "recollision" (operator of type $T^{\varepsilon}$ ). In the sequel, we shall systematically identify the generic term $\mathcal{T}(t)$, the associated collision sequence $\left\{\left(r_{k}, \ell_{k}\right)\right\}_{k=1}^{n}$, and the associated graph (see below).

It is useful to introduce the following graphical representation of the generic term $\mathcal{T}(t)$ in $(3.4)$, in the spirit of Feynman diagram expansions. We refer to figure 1 for an illustrative example in the particular case of the collision sequence

$$
\left\{\left(r_{k}, \ell_{k}\right)\right\}_{k=1}^{10}=\{(1,2),(1,2),(1,2),(2,3),(1,2),(3,4),(3,4),(3,2),(4,5),(4,5)\},
$$




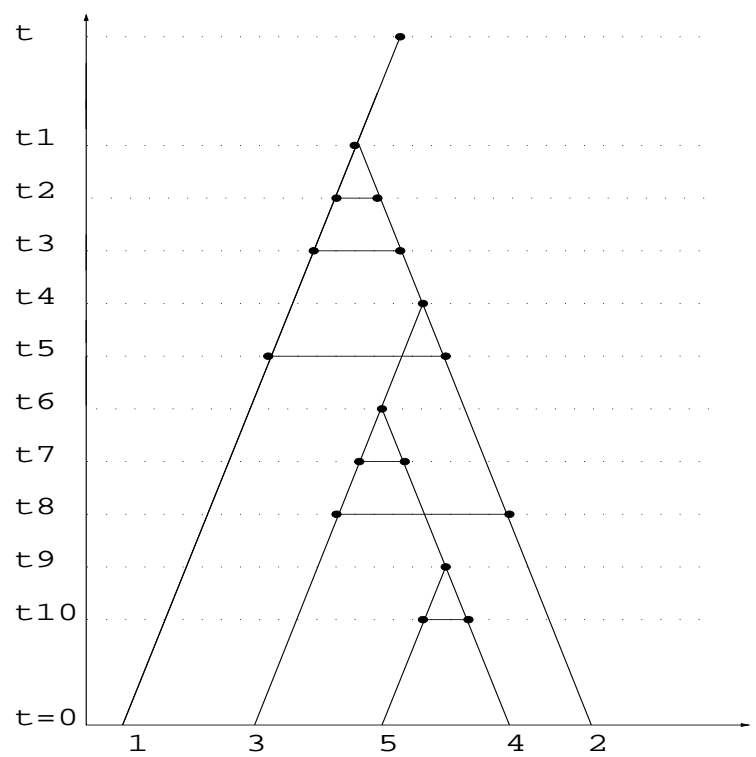

Figure 1: The graph associated with the collision sequence $\{(1,2),(1,2),(1,2),(2,3),(1,2),(3,4),(3,4),(3,2),(4,5),(4,5)\}$

for which $m=5$ (recall we restrict our attention to the study of $f_{1}^{N}$, i.e. the number of particles under consideration at the final time $t$ is one), and the total number of collision events of type $T^{\varepsilon}$ or $C^{\varepsilon}$ is $n=10$.

In figure 1 and as a general convention, the legs of the graph denote the particles themselves. Each leg represents one particle that is actually involved in the generic term (3.4). It carries the index of the corresponding particle. The nodes correspond to the creation of a particle, i.e. to a $C^{\varepsilon}$ operator. The horizontal segments correspond to a recollision, i.e. to a $T^{\varepsilon}$ operator. In both cases the node or the horizontal segment connect the two legs $r_{k}$ and $\ell_{k}$, corresponding to the particles actually involved in the associated collision event at time $t_{k}$. The straight lines between two successive collision times $t_{k}, t_{k+1}$ represent the free flight $S\left(t_{k}-t_{k+1}\right)$ entering the Duhamel expansion (3.4). Note that time runs backwards, from time $t$ on the top, to time 0 at the bottom.

To be complete, let us last mention a point of terminology: whenever an operator $C_{r_{k}, \ell_{k}}^{\varepsilon}$ is involved in (3.4), we shall say particle $\ell_{k}$ is a son of particle $r_{k}$, while $r_{k}$ is its father. For instance, in figure 1 , particle 3 is the son of particle 2 , etc. In the same vein, we shall typically call particle 2 an ancestor of particles 4 or 5 . Note this terminology again uses the fact that time is thought as running backwards is these diagrams, from time $t$ at the top to time 0 at the bottom. In this spirit, we shall conventionally say a collision occurring at time $t_{i}$ occurs before collision occuring at time $t_{j}$ whenever $t_{i}>t_{j}$, i.e. $i<j$ : the words "before" and "after" will be systematically used in reference to the indices of the collisional times, rather than in reference to the actual values of the latter.

Amongst all the indices $1, \ldots, n$ of the collision times $\left\{t_{k}\right\}_{k=1}^{n}$ we shall distinguish those times that correspond to the creation of a new particle. We shall denote these indices by $\left\{z_{p}\right\}_{p=2}^{m}$. In other words,

$z_{p}=j$ whenever particle $p$ has been created at time $t_{j}=t_{z_{p}}$.

In the example of figure 1 , we have $z_{2}=1, z_{3}=4, z_{4}=6, z_{5}=9$. We shall set $Z=\left\{z_{2}, \ldots, z_{m}\right\}$.

If $p$ is the index of a particle, we define the "cluster of $p$ " denoted by $C_{p}$, as being

$$
C_{p}=\{q \mid \text { particle } p \text { is an ancestor of particle } q\} \cup\{p\} .
$$

In other words, the set $C_{p}$ is the list of all descendants of particle $p$ (including $p$ ). In the example of figure 1 , we have $C_{1}=\{1,2,3,4,5\}$ (note the cluster of particle one always includes all the created particles), $C_{2}=$ $\{2,3,4,5\}, C_{3}=\{4,5\}, C_{4}=\{4,5\}, C_{5}=\{5\}$. Given $C_{p}$ we also denote by $E_{p}$ the set of indices of recollision times that involve one descendent of $p$ with one particle that is not a descendent of $p$, i.e. recollisions that are 
somehow "external" to the cluster $C_{p}$. In other words, we set

$$
E_{p}=\left\{j \notin Z \mid \text { collision number } j \text { involves particles } q_{1} \text { and } q_{2} \text { with } q_{1} \in C_{p} \text { but } q_{2} \notin C_{p}\right\} .
$$

In the example of figure 1 , we have $E_{1}=\emptyset$ (note $E_{1}$ is always void), $E_{2}=\{2,3,5\}, E_{3}=\{8\}, E_{4}=\{7\}$, $E_{5}=\{10\}$.

\subsection{Fourier transform of the distribution function}

In what follows, it will be more convenient to pass to the limit on the Fourier transform of the generic term $\mathcal{T}\left(t, x_{1}, v_{1}\right)$ given by (3.4). For that reason, we introduce several notations that appear on the Fourier side.

We define the Fourier transform of the $f_{k}^{0}$ 's as

$$
\widehat{f_{k}^{0}}(\xi ; \eta)=\int_{\mathbb{R}^{2 d k}} d x d v \mathrm{e}^{-i \xi \cdot x-i \eta \cdot v} f_{k}^{0}(x ; v), \quad f_{k}^{0}(x ; v)=\frac{1}{(2 \pi)^{2 d k}} \int_{\mathbb{R}^{2 d k}} d \xi d \eta \mathrm{e}^{+i \xi \cdot x+i \eta \cdot v} \widehat{f_{k}^{0}}(\xi ; \eta)
$$

with $\xi=\left(\xi_{1}, \ldots, \xi_{k}\right) \in \mathbb{R}^{d k}$ and similarly for $\eta$. A simple computation shows the operators $T^{\varepsilon}$ and $C^{\varepsilon}$, when written in Fourier variables, take the form

$$
\begin{aligned}
& \widehat{T}_{r, \ell}^{\varepsilon} \widehat{f_{k}^{0}}(\xi ; \eta)=\frac{-i}{\varepsilon^{1 / 2}} \sum_{\sigma= \pm 1} \sigma \int_{\mathbb{R}^{d}} d h \widehat{\phi}(h) \mathrm{e}^{i \frac{\sigma}{2} h \cdot\left(\eta_{\ell}-\eta_{r}\right)} \\
& \widehat{f_{k}^{0}}\left(\xi_{1}, \ldots, \xi_{r}-\frac{h}{\varepsilon}, \ldots, \xi_{\ell}+\frac{h}{\varepsilon}, \ldots \xi_{k} ; \eta_{1}, \ldots, \eta_{k}\right), \\
& \widehat{C}_{r, k+1}^{\varepsilon} \widehat{f_{k+1}^{0}}(\xi ; \eta)=\frac{-i}{\varepsilon^{d+1 / 2}} \sum_{\sigma= \pm 1} \sigma \int_{\mathbb{R}^{d}} d h \widehat{\phi}(h) \mathrm{e}^{-i \frac{\sigma}{2} h \cdot \eta_{r}} \\
& \widehat{f_{k+1}^{0}}\left(\xi_{1}, \ldots, \xi_{r}-\frac{h}{\varepsilon}, \ldots, \xi_{k}, \frac{h}{\varepsilon} ; \eta_{1}, \ldots, \eta_{k}, 0\right) .
\end{aligned}
$$

Similarly, the free streaming flow $S(t) f_{k}^{0}(x, v)=f_{k}^{0}(x-v t, v)$ is given in terms of Fourier transform by

$$
\widehat{S}(t) \widehat{f_{k}^{0}}(\xi ; \eta)=\widehat{f_{k}^{0}}(\xi ; \eta+t \xi) \text {. }
$$

Note the vector $(0, \ldots, 0,-h, 0, \ldots, 0,+h, 0, \ldots, 0)$, where the " $-h "$ is in position $r$, and the " $+h$ " is in position $\ell$, plays a particular role in formula (3.9) (it then should be seen as a vector in $\mathbb{R}^{d k}$ ) and in formula (3.10) (it then is a vector in $\left.\mathbb{R}^{d(k+1)}\right)$. As a consequence, it is natural to introduce the vectors $\theta_{r, \ell}(h) \in \mathbb{R}^{d m}$, defined as

$$
\theta_{r, \ell}(h)=(0, \ldots,-h, \ldots, h, \ldots, 0) \in \mathbb{R}^{d m}
$$

where $-h$ and $h$ are in the $r$-th and $\ell$-th position respectively. In other words, if for $p=1, \ldots, m$, the notation $e_{p}$ designates the natural projector defined by

$$
e_{p}: X=\left(x_{1}, \ldots, x_{m}\right) \in \mathbb{R}^{d m} \longmapsto e_{p}(X)=e_{p} \cdot X=x_{p} \in \mathbb{R}^{d},
$$

then $\theta_{r, \ell}$ is defined through

$$
e_{r}\left(\theta_{r, \ell}(h)\right)=-h, \quad e_{\ell}\left(\theta_{r, \ell}(h)\right)=+h, \quad \text { and } e_{p}\left(\theta_{r, \ell}(h)\right)=0 \text { whenever } p \neq r, \ell .
$$

In (3.13) and later, the two notations $e_{p}(X)$ and $e_{p} \cdot X$ are used indifferently. In a similar way, we also introduce the vectors $\theta_{k} \in \mathbb{R}^{m}$ defined as

$$
\theta_{k}=(0, \ldots,-1, \ldots, 1, \ldots, 0) \in \mathbb{R}^{m}
$$

where -1 and 1 are placed in the $r_{k}$ and $\ell_{k}$ positions respectively,

and $\left(r_{k}, \ell_{k}\right)$ is the pair of colliding particles at the collision time $t_{k}$. 
Note also that, setting

$$
\theta_{k}(h)=\theta_{r_{k}, \ell_{k}}(h),
$$

we have the identity

$$
\theta_{k}(h) \cdot \theta_{k^{\prime}}\left(h^{\prime}\right)=\left(h \cdot h^{\prime}\right)\left(\theta_{k} \cdot \theta_{k^{\prime}}\right) .
$$

In a sense, $\theta_{k}(h)$ is a multiplication of $\theta_{k}$ by $h$, so that we shall sometimes make the abuse of notation

$$
\theta_{k}(h)=\theta_{k} h .
$$

The conclusion is that, using the above notations, the generic term $\mathcal{T}\left(t, x_{1}, v_{1}\right)$ satisfies

$$
\begin{array}{r}
\widehat{\mathcal{T}}\left(t, \xi_{1}, \eta_{1}\right)=\varepsilon^{-d(m-1)-n / 2}(-i)^{n} \sum_{\sigma_{1}, \ldots \sigma_{n}= \pm 1} \prod_{j=1}^{n} \sigma_{j} \int_{0}^{t} d t_{1} \int_{0}^{t_{1}} d t_{2} \cdots \int_{0}^{t_{n-1}} d t_{n} \\
\int_{\mathbb{R}^{n d}} d h_{1} d h_{2} \cdots d h_{n}\left[\prod_{j=1}^{n} \widehat{\phi}\left(h_{j}\right)\right] \widehat{f_{m}^{0}}\left(\xi+\frac{H}{\varepsilon} ; \eta+t \xi+\frac{T}{\varepsilon}\right) \exp \left(i \frac{S_{\varepsilon}}{2 \varepsilon}\right),
\end{array}
$$

where $\xi=\left(\xi_{1}, 0, \ldots, 0\right) \in \mathbb{R}^{d m}, \eta=\left(\eta_{1}, 0, \ldots, 0\right) \in \mathbb{R}^{d m}$, and

$$
H=\sum_{j=1}^{n} \theta_{j} h_{j} \in \mathbb{R}^{d m}, \quad T=\sum_{j=1}^{n} t_{j} \theta_{j} h_{j} \in \mathbb{R}^{d m} .
$$

The phase $S_{\varepsilon}$ in (3.19) is given by

$$
S_{\varepsilon}=\sigma_{1} \theta_{1}\left(h_{1}\right) \cdot \eta_{\varepsilon}^{(1)}+\sigma_{2} \theta_{2}\left(h_{2}\right) \cdot \eta_{\varepsilon}^{(2)}+\cdots+\sigma_{n} \theta_{n}\left(h_{n}\right) \cdot \eta_{\varepsilon}^{(n)}
$$

where

$$
\begin{aligned}
& \eta_{\varepsilon}^{(1)}=\varepsilon\left[\eta+\left(t-t_{1}\right) \xi\right], \\
& \eta_{\varepsilon}^{(2)}=\varepsilon\left[\eta+\left(t-t_{2}\right) \xi\right]+\left(t_{1}-t_{2}\right) \theta_{1}\left(h_{1}\right), \\
& \ldots \\
& \eta_{\varepsilon}^{(n)}=\varepsilon\left[\eta+\left(t-t_{n}\right) \xi\right]+\sum_{r=1}^{n-1}\left(t_{r}-t_{n}\right) \theta_{r}\left(h_{r}\right) .
\end{aligned}
$$

Note that the structure of the graph identifying the term $\mathcal{T}$ under consideration, enters through the definition of $H, T$ and $S_{\varepsilon}$, namely through the form of the vectors $\theta_{j}$.

Remark 2. In the sequel, the variables $\left\{h_{k}\right\}_{k=1}^{n}$ are called the exchanged momenta (at the collision times $\left.\left\{t_{k}\right\}_{k=1}^{n}\right)$.

Remark 3. The arguments of $\widehat{f_{m}^{0}}$ in (3.19) may as well be written

$$
\widehat{f_{m}^{0}}\left(\xi+\frac{H}{\varepsilon} ; \eta+t \xi+\frac{T}{\varepsilon}\right)=\widehat{f_{m}^{0}}\left(\xi_{1}+\frac{H_{1}}{\varepsilon}, \frac{H_{2}}{\varepsilon}, \ldots, \frac{H_{m}}{\varepsilon} ; \eta_{1}+t \xi_{1}+\frac{T_{1}}{\varepsilon}, \frac{T_{2}}{\varepsilon}, \ldots, \frac{T_{m}}{\varepsilon}\right),
$$

up to denoting $H_{p}=e_{p} \cdot H$ and $T_{p}=e_{p} \cdot T$. With these notations, an obvious yet very important property is the following

$$
\sum_{p=1}^{m} H_{p}=0, \quad \sum_{p=1}^{m} T_{p}=0 .
$$

These two identities come from the fact that for each $j$, the vector $h_{j}$ only appears in the two factors $H_{r_{j}}=*-h_{j}$ and $H_{\ell_{j}}=*+h_{j}$, where the “*”'s denotes some functions that depend on the $h_{k}$ 's for $k \neq j$ only. The two opposite signs give the result. 
Remark 4. The arguments of $\widehat{f_{m}^{0}}$ in (3.19) can be recovered upon looking at the exchanged momenta in the graph. For instance, in the case of figure 2, we have

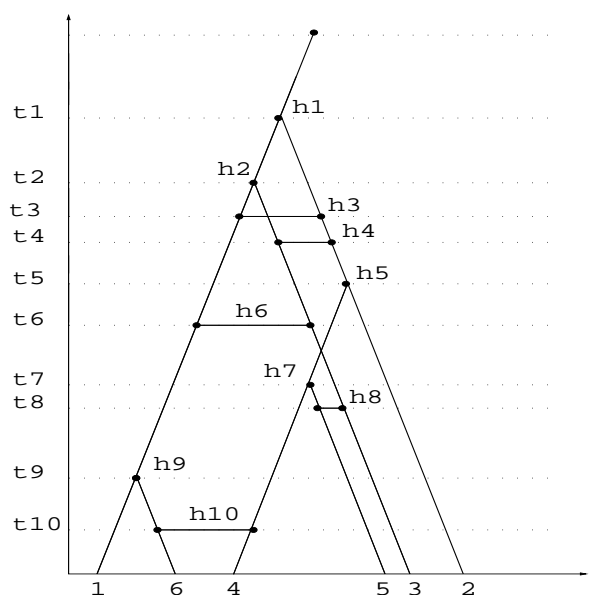

Figure 2: The exchanged momenta

$$
\xi+\frac{H}{\varepsilon}=\left(\begin{array}{c}
\xi_{1}+\varepsilon^{-1}\left(-h_{1}-h_{2}-h_{3}-h_{6}-h_{9}\right) \\
\varepsilon^{-1}\left(h_{1}+h_{3}-h_{4}-h_{5}\right) \\
\varepsilon^{-1}\left(h_{2}+h_{4}+h_{6}-h_{8}\right) \\
\varepsilon^{-1}\left(h_{5}-h_{7}-h_{10}\right) \\
\varepsilon^{-1}\left(h_{7}+h_{8}\right) \\
\varepsilon^{-1}\left(h_{9}+h_{10}\right)
\end{array}\right)
$$

and

$$
\eta+t \xi+\frac{T}{\varepsilon}=\left(\begin{array}{c}
\eta_{1}+t \xi_{1}+\varepsilon^{-1}\left(-h_{1} t_{1}-h_{2} t_{2}-h_{3} t_{3}-h_{6} t_{6}-h_{9} t_{9}\right) \\
\varepsilon^{-1}\left(h_{1} t_{1}+h_{3} t_{3}-h_{4} t_{4}-h_{5} t_{5}\right) \\
\varepsilon^{-1}\left(h_{2} t_{2}+h_{4} t_{4}+h_{6} t_{6}-h_{8} t_{8}\right) \\
\varepsilon^{-1}\left(h_{5} t_{5}-h_{7} t_{7}-h_{10} t_{10}\right) \\
\varepsilon^{-1}\left(h_{7} t_{7}+h_{8} t_{8}\right) \\
\varepsilon^{-1}\left(h_{9} t_{9}+h_{10} t_{10}\right)
\end{array}\right)
$$

In that case, we also have $z_{2}=1, z_{3}=2, z_{4}=5, z_{5}=7, z_{6}=9$, as well as $C_{1}=\{1,2,3,4,5,6\}, C_{2}=\{2,4,5\}$, $C_{3}=\{3\}, C_{4}=\{4,5\}, C_{5}=\{5\}, C_{6}=\{6\}$, and last $E_{1}=\emptyset, E_{2}=\{3,4,8,10\}, E_{3}=\{4,6,8\}, E_{4}=\{8,10\}$, $E_{5}=\{8\}, E_{6}=\{10\}$.

\section{Organizing the graphs}

At the heuristic level, we know from [BCEP1] that there is only one class of $\mathcal{T}(t)$ 's, i.e. only one class of graphs or collision sequences $\left\{\left(r_{k}, \ell_{k}\right)\right\}_{k=1}^{n}$, that give rise to a non vanishing contribution in the limit $\varepsilon \rightarrow 0$. This is the class of collision-recollision sequences, i.e. graphs of the form

$$
\{\underbrace{\left(r_{1}\right)=\left(r_{3}, \ell_{3}\right)=\left(r_{3}, 3\right),\left(r_{4}, \ell_{4}\right)=\left(r_{3}, 3\right)}_{\ldots \ldots,(\underbrace{\left(r_{2 j-1}, \ell_{2 j-1}\right)=\left(r_{2 j-1}, j+1\right),\left(r_{2 j}, \ell_{2 j}\right)=\left(r_{2 j-1}, j+1\right)}, \ldots . .},
$$

Equivalently, collision-recollision sequences correspond to contributions of the form

$$
\underbrace{S C_{1,2}^{\varepsilon} S T_{1,2}^{\varepsilon}} \underbrace{S C_{r_{3}, 3}^{\varepsilon} S T_{r_{3}, 3}^{\varepsilon}} \ldots \underbrace{S C_{r_{2 j-1}, j+1}^{\varepsilon} S T_{r_{2 j-1}, j+1}^{\varepsilon}} \ldots S f_{m}^{0},
$$


where particle 2 is created from particle 1 (at time $t_{1}$ ), then immediately recollides with particle 1 (at time $t_{2}$ ), next particle 3 is created from particle $r_{3}=1$ or 2 (at time $t_{3}$ ), then immediately recollides with particle $r_{3}$ (at time $t_{4}$ ), etc. Such a graph is illustrated in figure 3 , in the case of the specific sequence $\{(1,2),(1,2),(2,3),(2,3),(2,4),(2,4),(1,5),(1,5)\}$.

We actually proved in [BCEP1] that the subseries of $f_{1}^{N}(t)$ that is constituted of all collision-recollision sequences, namely

$$
\begin{aligned}
g^{N}(t):= & \sum_{k=0}^{N-1} \sum_{1 \leq r_{1} \leq 1} \sum_{1 \leq r_{2} \leq 2} \ldots \sum_{1 \leq r_{k} \leq k+1} \int\left[S C_{r_{1}, 2}^{\varepsilon} S T_{r_{1}, 2}^{\varepsilon}\right]\left[S C_{r_{2}, 3}^{\varepsilon} S T_{r_{2}, 3}^{\varepsilon}\right] \\
& \cdots\left[S C_{r_{k}, k+1}^{\varepsilon} S T_{r_{k}, k+1}^{\varepsilon}\right] S f_{0}^{\otimes(k+1)}
\end{aligned}
$$

indeed converges, uniformly for short times, towards $f(t)$, solution to the Boltzmann equation. In this perspective, our main Theorem is proved once we are able to prove that all other graphs are vanishing as $\varepsilon \rightarrow 0$. In order to do so, we need to classify the graphs in an appropriate fashion.

Definition 1. A recollision $T_{r, \ell}^{\varepsilon}$ is called proper whenever it involves two particles $r$ and $\ell, r<\ell$, such that $\ell$ is a son of $r$.

Definition 2. A graph is called a right graph (see figure 3) whenever the following conditions are fulfilled:

(1) $\frac{n}{2}=m-1$, or, in other words $n-m-1=m-1$ (the number of "creations" $C^{\varepsilon}$ equals that of "recollisions"
$T^{\varepsilon}$ ).

(2) for any creation $C_{r, \ell}^{\varepsilon}$, the graph also involves exactly one recollision $T_{r, \ell}^{\varepsilon}$ between the same particles,

(3) all creation-recollision events $C_{r, \ell}^{\varepsilon}$ and $T_{r, \ell}^{\varepsilon}$ involving the same two particles are consecutive (i.e. the recollision $T_{r, \ell}^{\varepsilon}$ occurs at the collision time $t_{j+1}$ whenever the creation $C_{r, \ell}^{\varepsilon}$ occurs at the collision time $\left.t_{j}\right)$.

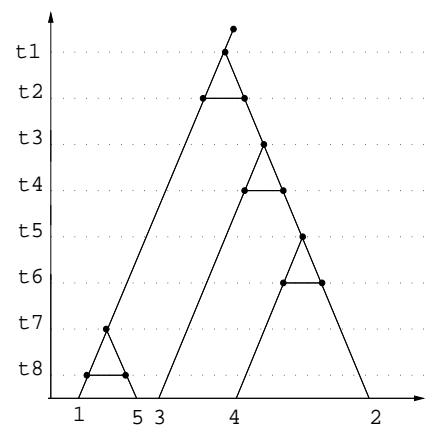

Figure 3: A collision-recollision sequence 
Definition 3. A graph that satisfies the above properties (1) and (2) without satisfying property (3) is called right non-ordered graph (see figure 4).

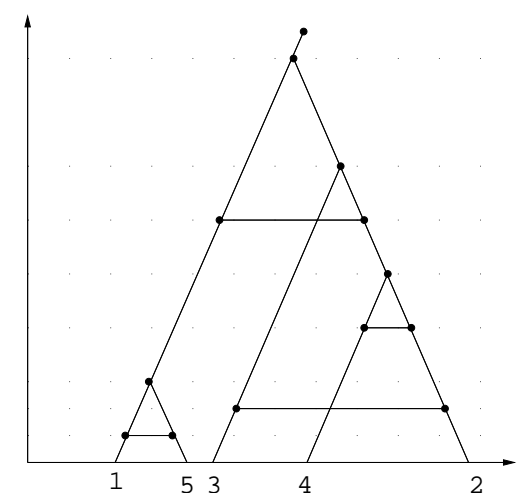

Figure 4: A right non-ordered graph

Definition 4. A graph that is neither right, nor right non-ordered, is called a wrong graph.

Remark 5. In that language, the subseries $g^{N}(t)$ in (4.2) is made up of all right graphs.

With these conventions, and on the basis what we proved in [BCEP1], we arrive at the

Proposition 1. In order to prove our main Theorem, it suffices to prove that the generic term $\widehat{\mathcal{T}}\left(t, \xi_{1} ; \eta_{1}\right)$ given by (3.19) goes to zero uniformly with $\xi_{1}$ and $\eta_{1}$ as $\varepsilon \rightarrow 0$, whenever the associated graph is either right non-ordered, or wrong.

The remainder part of this text is devoted to the proof of Proposition 1.

\section{Three basic Lemmas}

We give here three Lemmas which are the basic ingredients to establish the desired Proposition 1, when conveniently combined with a stationary phase analysis we provide later in this text.

The basic observation is the following. The arguments of $\widehat{f}_{m}$ in (3.19) (see Remarks 3 and 4 ), namely

$$
\widehat{f_{m}^{0}}\left(\xi+\frac{H}{\varepsilon} ; \eta+t \xi+\frac{T}{\varepsilon}\right)=\widehat{f_{m}^{0}}\left(\xi_{1}+\frac{H_{1}}{\varepsilon}, \frac{H_{2}}{\varepsilon}, \ldots, \frac{H_{m}}{\varepsilon} ; \eta_{1}+t \xi_{1}+\frac{T_{1}}{\varepsilon}, \frac{T_{2}}{\varepsilon}, \ldots, \frac{T_{m}}{\varepsilon}\right)
$$

involve the momenta $\left\{H_{p}\right\}_{p=2}^{m}$. The key point is, one can express the creation momenta $\left\{h_{z_{p}}\right\}_{p=2}^{m}$, i.e. the exchanged momenta at the creation times $\left\{t_{z_{p}}\right\}_{p=2}^{m}$, as an explicit function of the arguments $\left\{H_{p}\right\}_{p=2}^{m}$, and $\left\{h_{j}\right\}_{j \notin Z}$. 
Quantitatively, the statement is the following

Lemma 1. For any particle $p=2, \ldots, m$, the following identity holds true

$$
h_{z_{p}}=\sum_{q \in C_{p}} H_{q}+\sum_{j \in E_{p}} \pi_{p, j} h_{j},
$$

where $\pi_{p, j}=+1$ if $j$ is the collision index between a particle $q \in C_{p}$ and $r \notin C_{q}$ with $r>q$, and $\pi_{p, j}=-1$ when $r<q$ (see figure 5)
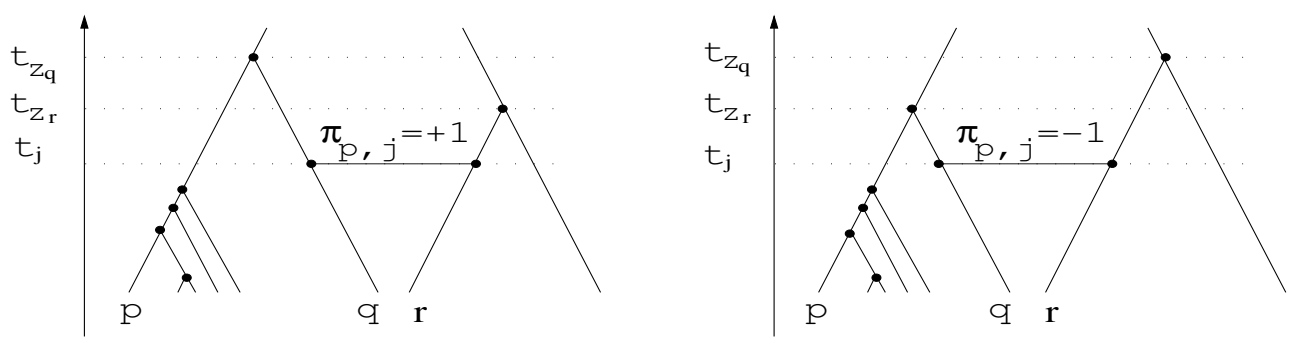

Figure 5: Value of $\pi_{p, j}$

\section{Proof of Lemma 1:}

Since

$$
\theta_{i} h_{i} \cdot e_{p}= \begin{cases}-h_{i} & \text { if } p=r_{i} \\ +h_{i} & \text { if } p=\ell_{i} \\ 0 & \text { otherwise }\end{cases}
$$

we have the first stage identity

$$
H_{p}=h_{z_{p}}-\sum_{q \in \mathcal{S}_{p}} h_{z_{q}}-\sum_{\ell \in \mathcal{R}_{p}} \tilde{\pi}_{p, \ell} h_{\ell},
$$

where $\mathcal{S}_{p}$ is the set of particles directly generated by $p$ (the sons of $p$ ), and $\mathcal{R}_{p}$ is the set of recollisions of particle $p$, namely $\mathcal{R}_{p}=\left\{j \mid\right.$ particle $p$ recollides at time $\left.t_{j}\right\}$. Last, $\tilde{\pi}_{p, \ell}$ is the sign of the collision with respect to particle $p$, i.e. $\tilde{\pi}_{p, \ell}=+1$ if the particle which recollides with $p$ at time $t_{\ell}$, say particle $r$, has been created after $p$, namely $z_{p}<z_{r}$, and $\tilde{\pi}_{p, \ell}=-1$ otherwise.

As a consequence, we directly obtain

$$
h_{z_{p}}=H_{p}+\sum_{\ell \in \mathcal{R}_{p}} \tilde{\pi}_{p, \ell} h_{\ell}+\sum_{p_{1} \in \mathcal{S}_{p}} h_{z_{p_{1}}} .
$$

The idea now is to iterate (5.3) up to the next "generation". Namely, we may write

$$
\begin{aligned}
h_{z_{p}}= & \left(H_{p}+\sum_{\ell \in \mathcal{R}_{p}} \tilde{\pi}_{p, \ell} h_{\ell}\right)+\sum_{p_{1} \in \mathcal{S}_{p}}\left(H_{p_{1}}+\sum_{\ell \in \mathcal{R}_{p_{1}}} \tilde{\pi}_{p_{1}, \ell} h_{\ell}+\sum_{p_{2} \in \mathcal{S}_{p_{1}}} h_{z_{p_{2}}}\right) \\
= & \left(H_{p}+\sum_{\ell \in \mathcal{R}_{p}} \tilde{\pi}_{p, \ell} h_{\ell}\right)+\sum_{p_{1} \in \mathcal{S}_{p}}\left(H_{p_{1}}+\sum_{\ell \in \mathcal{R}_{p_{1}}} \tilde{\pi}_{p_{1}, \ell} h_{\ell}\right) \\
& +\sum_{p_{1} \in \mathcal{S}_{p}} \sum_{p_{2} \in \mathcal{S}_{p_{1}}}\left(H_{p_{2}}+\sum_{\ell \in \mathcal{R}_{p_{2}}} \tilde{\pi}_{p_{1}, \ell} h_{\ell}+\sum_{p_{3} \in \mathcal{S}_{p_{2}}} h_{z_{p_{3}}}\right)
\end{aligned}
$$


and continue up to the elimination of the last $h_{j}, j \in Z$, i.e. up to the last generation. The result is

$$
h_{z_{p}}=\sum_{q \in C_{p}} H_{q}+\sum_{q \in C_{p}} \sum_{\ell \in \mathcal{R}_{q}} \tilde{\pi}_{q, \ell} h_{\ell} .
$$

Finally (5.1) is a consequence of the following fact. If $j$ is the index of an internal recollision for $p$, i.e. of a collision between $p$ and $q$ with $q \in C_{p}$, then the exchanged momentum $h_{j}$ appears exactly two times in the last sum of (5.5), yet with opposite signs (see also Remark 3): they sum up to zero. Hence the only terms surviving in the last sum of (5.5) are those involving an external collision of the cluster of $p$. They are indexed by the elements of $E_{p}$. Reminding the definition of $\pi_{p, j}$, Lemma 1 is now proved.

We next give two Lemmas which characterize, in the particular case when $m-1=n / 2$, i.e. in the case when the number $m-1$ of creations equals the number $n-(m-1)$ of recollisions, the graphs that are either wrong or right non-ordered.

\section{Lemma 2. (Right non-ordered graphs)}

Let $m>2$. Assume the graph $\mathcal{T}(t)$ is right non-ordered, and hence $m-1=n / 2$. For each $r=2, \ldots, m$, denote by $i(r)$ the (unique) recollision index of particle $r$ with the particle which created it (i.e. his father).

Then the graph $\mathcal{T}(t)$ involves at least two particles $p$ and $q, p<q$, such that

$$
z_{p}<z_{q}<i(p)
$$

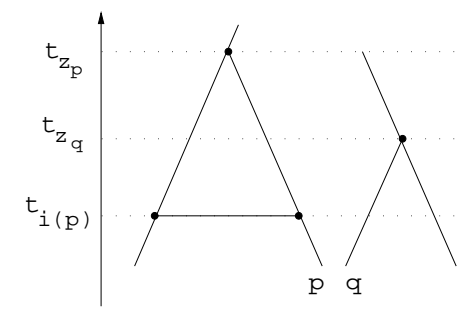

Figure 6: Illustration of Lemma 2

Remark 6. In other words, the fact that $\mathcal{T}(t)$ is non-ordered implies there are two particles $p$ and $q$ such that particle $q$ is created after $p$, yet before particle $p$ recollides with its father.

Such a situation is excluded in the case of right graphs: there, each recollision has to immediately follow the associated creation.

Lemma 3. (Wrong graphs with $m-1=n / 2$ )

Let $m>2$ and $m-1=\frac{n}{2}$. Assume $\mathcal{T}(t)$ is a wrong graph. Then, the graph $\mathcal{T}(t)$ involves at least one particle $p$ for which (here $\left|E_{p}\right|$ denotes the cardinality of $E_{p}$ )

i) $\left|E_{p}\right| \geq 2$,

ii) $\exists i \in E_{p}$ for which $\pi_{p, i}=-1$.

Remark 7. In other words, the fact $\mathcal{T}(t)$ is wrong implies there is a particle $p$ such that the set formed by all its descendants is involved at least twice in collisions with particles that do not descend from $p$. Besides, at least one amongst these collisions involves a descendent of $p$ and another particle $q$ (which is not a descendent of $p$ ), such that particle $q$ is created before $p$. (Recall in passing that in the chosen terminology, $p$ is considered a descendent of himself). 


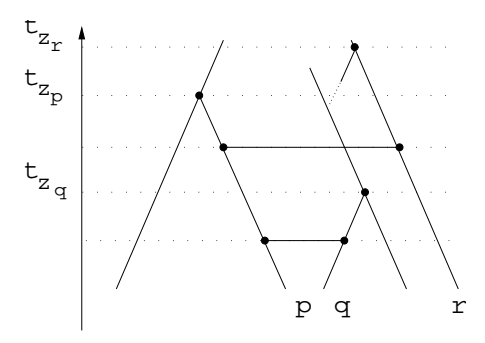

Figure 7: Illustration of Lemma 3

\section{Proof of Lemma 2:}

Since the graph is right non ordered, there is a particle $p$, created at time $t_{z_{p}}$ and recolliding with its father at time $t_{i(p)}$, such that at least one other collision event occurs between these two times $t_{z_{p}}$ and $t_{i(p)}$ (the time $t_{i(p)}$ is well-defined for any value of $p$ by the very definition of right non-ordered graphs, and the wrong ordering gives the second part of the statement). If this distinguished collision event corresponds to the creation of a particle $q$, which necessarily occurs at time $t_{z_{q}}$, then the lemma is proved since $z_{p}<z_{q}<i(p)$. If the event is a recollision between a particle $q$, with associated creation index $z_{q}$, and its father, then the recollision necessarily happens at time $t_{i(q)}$. There are now two possibilities: either particle $q$ has been created after particle $p$, and then the lemma is proved, or it has been created before, so that $z_{q}<z_{p}<i(q)$ and the lemma is proved with $q$ and $p$ interchanged.

\section{Proof of Lemma 3:}

When $m=3$ the Lemma is easily proved by direct inspection. It is indeed an easy exercise to write down the explicit list of the 10 wrong graphs with $m=3$ (and $n=4$ ).

When $m>3$ we prove the Lemma by induction, assuming it is true for the value $m-1$. We distinguish three cases, depending on whether $\left|E_{m}\right| \geq 2,\left|E_{m}\right|=1$ or $\left|E_{m}\right|=0$.

Case (i) $-\left|E_{m}\right| \geq 2$.

In that case we necessarily have $\pi_{m, i}=-1$ for any collision time $t_{i}$ for which particle $m$ is involved. This simply comes from the fact $m$ is the last created particle.

The induction is proved in the case $\left|E_{m}\right| \geq 2$.

Case (ii) $-\left|E_{m}\right|=0$.

In that case we first build up, starting from the graph $\mathcal{T}(t)$, a lower order graph, say $\widetilde{\mathcal{T}}(t)$, that involves $m-1$ particles, $m-2$ creations, and $m-2$ recollisions. To do so, we pick up a collision time $t_{i}$ that corresponds to a recollision. To fix the notations, let us say the collision time $t_{i}$ involves the two particles $p$ and $q$ with $q<p$ (hence $p>1$ ). The new graph $\widetilde{\mathcal{T}}(t)$ is then simply obtained upon erasing the horizontal segment corresponding to the recollision occuring at time $t_{i}$, together with the whole leg corresponding to particle $m$. This is illustrated in figure 8 .

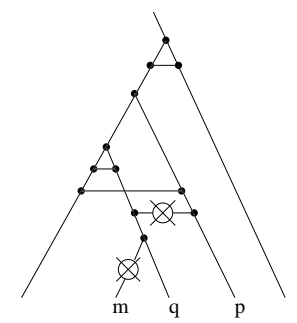

Figure 8: Building up the new graph $\widetilde{\mathcal{T}}(t)$ starting from $\mathcal{T}(t)$ - the case $\left|E_{m}\right|=0$

If the graph $\widetilde{\mathcal{T}}(t)$ obtained in this way is wrong, we may use the induction hypothesis. 
If $\widetilde{\mathcal{T}}(t)$ is right or right non-ordered, then particle $p$ is involved in at least two external recollisions inside the larger graph $\mathcal{T}(t)$ : $(a)$ the proper one, namely the one that involves $p$ and its father, which happens at time $t_{i(p)}$ (this collision necessarily happens because $p>1$ ). We have $\pi_{p, i(p)}=-1$. (b) The collision with particle $q$, which occurs at time $t_{i}$, say. It satisfies $\pi_{p, i}=-1$ as well (simply because $q<p$ ).

In any circumstance, the induction is proved in the case $\left|E_{m}\right|=0$.

Case (iii) $-\left|E_{m}\right|=1$.

In order to fix the ideas, let us say $E_{m}=\{i\}$.

In that situation, we may build up again a lower order graph $\widetilde{\mathcal{T}}(t)$ (we use the same symbol not to overweight notations) which involves $m-1$ particles, $m-2$ creations, and $m-2$ recollisions. It is obtained upon erasing the horizontal segment corresponding to the recollision occurring at time $t_{i}$, together with the whole leg corresponding to particle $m$. This construction is relevant since particle $m$ is only involved in the collision with index $i$, by our very assumption.

There are now two subcases.

Let us assume first $i$ is the index of a proper recollision, see figure 9 .

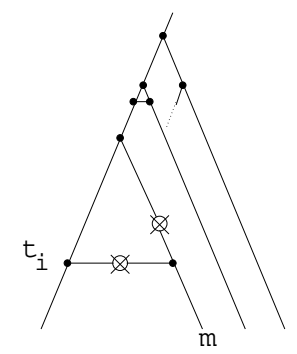

Figure 9: Building up $\widetilde{\mathcal{T}}(t)$ starting from $\mathcal{T}(t)$ : the case when $\left|E_{m}\right|=1$ and $i$ is a proper recollision

If the lower order graph $\widetilde{\mathcal{T}}(t)$ is right, resp. right non-ordered, then the full graph $\mathcal{T}(t)$ is right, resp. right non-ordered as well. This is not possible (we assumed $\mathcal{T}(t)$ is wrong). Hence $\widetilde{\mathcal{T}}(t)$ is necessarily wrong. We are thus in position to use the induction hypothesis.

Second, in the case when $i$ is the index of a non proper recollision, see figure 10 and figure 11, we may assume, to fix notations, this recollision involves particles $m$ and $p$, where $p$ is not $m$ 's father.

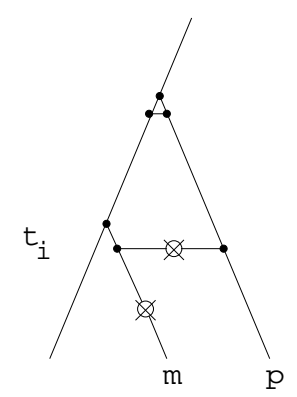

Figure 10: Building up $\widetilde{\mathcal{T}}(t)$ starting from $\mathcal{T}(t)$ : the case when $\left|E_{m}\right|=1$ and $i$ is not a proper recollision $(p>1)$

If $\widetilde{\mathcal{T}}(t)$ is wrong, we may use the induction hypothesis.

If $\widetilde{\mathcal{T}}(t)$ is right, or right non-ordered, the situation is as follows. On the one hand, if $p>1$, see figure 10 , then particle $p$ is involved in at least two external recollisions inside the larger graph $\mathcal{T}(t)$, namely the proper one, which occurs at the collision time $t_{i(p)}$, and the collision occuring at time $t_{i}$. Naturally, we have $\pi_{p, i(p)}=-1$.

If $p=1$, see figure 11, we have to modify the argument a bit since $p=1$ does not have any father. However, since particle 1 is not $m$ 's father ( $i$ is the index of a non-proper recollision), there exists a particle $q \neq 1$ which 


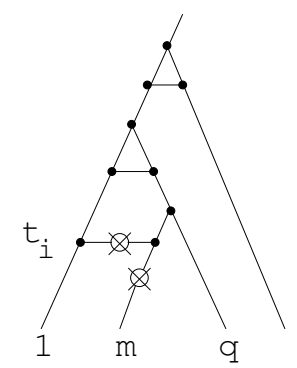

Figure 11: Building up $\widetilde{\mathcal{T}}(t)$ starting from $\mathcal{T}(t)$ : the case when $\left|E_{m}\right|=1$ and $i$ is not a proper recollision $(p=1)$

is an ancestor of $m$. As a consequence, the set $E_{q}$ obtained from the larger graph $\mathcal{T}(t)$ contains at least the index $i$, plus the proper recollision that occurs at time $t_{i(q)}$. Naturally, we have $\pi_{q, i(q)}=-1$.

This finishes the induction in the case $\left|E_{m}\right|=1$.

Lemma 3 is proved.

\section{Graphs for which $m-1<n / 2$}

In this section, we analyze in formula (3.19) the contribution of graphs for which $m-1<n / 2$ (i.e. for which the number of recollisions is larger than the number of creation). We prove these graphs vanish as $\varepsilon \rightarrow 0$.

This necessitates to prove the integral over $t_{1}, \ldots, t_{n}, h_{1}, \ldots, h_{n}$ in $(3.19)$ has size smaller than $\varepsilon^{+d(m-1)+n / 2}$ as $\varepsilon \rightarrow 0$. This piece of information is obtained upon analyzing the fast oscillations induced by the phase factor $\exp \left(i S_{\varepsilon} / 2 \varepsilon\right)$ in $(3.19)$, in combination with an appropriate stationary phase argument.

The main result of this paragraph is the following

Proposition 2. Let $\mathcal{T}(t)$ be a graph such that $m-1<n / 2$. Then, under the smoothness assumptions of our main theorem, there is a constant $C=C\left(d, m, n, \phi, f_{0}, t\right)$, which depends on all the mentioned arguments but not on $\varepsilon$, such that

$$
\left\|\widehat{\mathcal{T}}\left(t, \xi_{1} ; \eta_{1}\right)\right\|_{L^{\infty}\left(\mathbb{R}^{d} \times \mathbb{R}^{d}\right)} \leq C \varepsilon^{n / 2-(m-1)} \underset{\varepsilon \rightarrow 0}{\longrightarrow} 0 .
$$

Remark 8. The dependence of $C$ upon the various parameters in Proposition 2 is of the form

$$
C \leq c(d)^{n}\left(\|\widehat{\phi}\|_{L_{1}}+\|\phi\|_{L_{1}}\right)^{n} \mathcal{N}_{\alpha}\left(f_{0}\right)^{m} \frac{t^{m-1}}{(m-1) !}
$$

where $c(d)$ only depends on the dimension.

Remark 9. In this part of the proof we do not require the assumption $\widehat{\phi}(0)=0$.

The remainder part of this paragraph is devoted to the proof of Proposition 2, which is organized into four steps.

First step: isolating the quadratic part of the phase factor $S_{\varepsilon}$ in $\widehat{\mathcal{T}}\left(t, \xi_{1}, \eta_{1}\right)$

We start from equation (3.19), which yields the value of $\widehat{\mathcal{T}}\left(t, \xi_{1}, \eta_{1}\right)$. It provides the estimate

$$
\begin{aligned}
\left|\widehat{\mathcal{T}}\left(t, \xi_{1}, \eta_{1}\right)\right| \leq \varepsilon^{-d(m-1)-n / 2} \sum_{\sigma_{1}, \ldots, \sigma_{n}= \pm 1} \int_{0}^{t} d t_{1} \int_{0}^{t_{1}} d t_{2} \cdots \int_{0}^{t_{n-1}} d t_{n} \\
\left|\int_{\mathbb{R}^{n d}} d h_{1} d h_{2} \cdots d h_{n} \prod_{j=1}^{n} \widehat{\phi}\left(h_{j}\right) \widehat{f_{m}^{0}}\left(\xi+\frac{H}{\varepsilon} ; \eta+t \xi+\frac{T}{\varepsilon}\right) \exp \left(i \frac{S_{\varepsilon}}{2 \varepsilon}\right)\right|,
\end{aligned}
$$


where $H$ and $T$ are defined in (3.20), and the phase factor is

$$
\begin{aligned}
S_{\varepsilon}= & \varepsilon \sum_{j=1}^{n} \sigma_{j} \theta_{j} h_{j} \cdot\left(\eta+\left(t-t_{j}\right) \xi\right)+ \\
& \sigma_{2} \theta_{2} h_{2} \cdot \theta_{1} h_{1}\left(t_{1}-t_{2}\right)+ \\
& \sigma_{3} \theta_{3} h_{3} \cdot\left(\theta_{1} h_{1}\left(t_{1}-t_{3}\right)+\theta_{2} h_{2}\left(t_{2}-t_{3}\right)\right)+ \\
& \cdots \\
& \sigma_{n} \theta_{n} h_{n} \cdot\left(\theta_{1} h_{1}\left(t_{1}-t_{n}\right)+\theta_{2} h_{2}\left(t_{2}-t_{n}\right)+\cdots+\theta_{n-1} h_{n-1}\left(t_{n-1}-t_{n}\right)\right)
\end{aligned}
$$

For later convenience, it is natural to introduce the time differences

$$
s_{j}=t_{j-1}-t_{j} \quad(j=2, \ldots, n),
$$

and express the phase in these new variables. With these notations, we recover

$$
\begin{aligned}
& \left|\widehat{\mathcal{T}}\left(t, \xi_{1}, \eta_{1}\right)\right| \leq \varepsilon^{-d(m-1)-n / 2} \sum_{\sigma_{1}, \ldots, \sigma_{n}= \pm 1} \int_{0}^{t} d t_{1} \int_{0}^{t_{1}} d t_{2} \int_{0}^{t_{2}} d t_{3} \cdots \int_{0}^{t_{n-1}} d t_{n} \\
& \left|\int_{\mathbb{R}^{n d}} d h_{1} d h_{2} \cdots d h_{n} \prod_{j=1}^{n} \widehat{\phi}\left(h_{j}\right) \widehat{f_{m}^{0}}\left(\xi+\frac{H}{\varepsilon} ; \eta+t \xi+\frac{T}{\varepsilon}\right) \exp \left(i \frac{L}{2}\right) \exp \left(i \frac{Q}{2 \varepsilon}\right)\right| .
\end{aligned}
$$

Here, $Q$ denotes the part of $S_{\varepsilon}$ which is quadratic in the $\left\{h_{k}\right\}_{k=1}^{n}$ 's. This is the important term. Its explicit value is

$$
\begin{aligned}
Q= & s_{2} \theta_{1} h_{1} \cdot\left(\sigma_{2} \theta_{2} h_{2}+\sigma_{3} \theta_{3} h_{3}+\cdots+\sigma_{n} \theta_{n} h_{n}\right)+ \\
& s_{3}\left(\theta_{1} h_{1}+\theta_{2} h_{2}\right) \cdot\left(\sigma_{3} \theta_{3} h_{3}+\cdots+\sigma_{n} \theta_{n} h_{n}\right)+ \\
& \cdots \\
& s_{n}\left(\theta_{1} h_{1}+\theta_{2} h_{2}+\cdots+\theta_{n-1} h_{n-1}\right) \cdot \sigma_{n} \theta_{n} h_{n}
\end{aligned}
$$

On the other hand, $L$ is the part of $S_{\varepsilon}$ which is linear in the $\left\{h_{k}\right\}_{k=1}^{n}$ 's. Its value is essentially irrelevant. However, for sake of completeness, we give

$$
L=\sigma_{1} \theta_{1} h_{1} \cdot\left(\eta+\left(t-t_{1}\right) \xi\right)+\sum_{j=2}^{n} \sigma_{j} \theta_{j} h_{j} \cdot\left(\eta+\left(t-s_{1}-\cdots-s_{j}\right) \xi\right) .
$$

\section{Second step: separating the fast and slow variables}

Now we want to perform the integrals with respect to $d h_{j}$, exploiting in $(6.3)$ the oscillatory factor $\exp (i Q /(2 \varepsilon))$. However, since $\widehat{f_{m}^{0}}$ depends on the "fast" variables $H_{j} / \varepsilon$, we need first to rescale $H$ by $\varepsilon$, while carefully separating the $H$ dependence of the phase factors. This is where Lemma 1 plays a crucial role as we now show.

To be more specific, we split the $\left\{h_{k}\right\}_{k=1}^{n}$ 's into the two sets of variables

$$
\left\{h_{k}\right\}_{k=1}^{n}=\left\{h_{k}\right\}_{k \in Z} \cup\left\{h_{k}\right\}_{k \notin Z},
$$

i.e. we separate the exchanged momenta into those associated with a recollision event, and those associated with a creation event. Since the creation momenta $\left\{h_{k}\right\}_{k \in Z}$ are essentially related with the $\left\{H_{p}\right\}_{p=2}^{m}$ 's, we also change variables

$$
\left\{h_{k}\right\}_{k \in Z} \mapsto\left\{H_{p}\right\}_{p=2}^{m}
$$

in (6.3). Our new integration variables in (6.3) are thus the $\left(\left\{h_{k}\right\}_{k \notin Z},\left\{H_{p}\right\}_{p=2}^{m}\right)$ 's. The reader should be cautious about the fact variable $H_{1}$ is put apart here, since particle 1 does not stem from any creation event. Yet $H_{1}$ is anyhow recovered from the $\left\{H_{p}\right\}_{p=2}^{m}$ 's through formula $\sum_{i=1}^{m} H_{i}=0$ (see remark 3 and equation (3.24)). On the other hand, thanks to Lemma 1, we have

$$
h_{z_{p}}=\sum_{q \in C_{p}} H_{q}+\sum_{j \in E_{p}} \pi_{p, j} h_{j}
$$


and the second sum only involves the $\left\{h_{j}\right\}_{j \notin Z}$ 's, while the first sum involves a triangular structure

$$
\sum_{q \in C_{p}} H_{q}=H_{p}+\sum_{q \in C_{p}, q>p} H_{q}
$$

For this reason, the mapping (6.6) is one-to-one, and it has Jacobian +1 .

We are now in position to give expressions for $\widehat{\mathcal{T}}(t)$, and for the fast phase $Q$, where the role of the $\left\{h_{k}\right\}_{k \notin Z}$ 's and that of the $\left\{H_{p}\right\}_{p=2}^{m}$ 's are clearly sorted out.

First, the definition of $H=\sum_{j=1}^{n} \theta_{j} h_{j}$ provides the equality

$$
\theta_{1} h_{1}+\cdots+\theta_{j-1} h_{j-1}=H-\left(\theta_{j} h_{j}+\cdots+\theta_{n} h_{n}\right) .
$$

From this we deduce a more symmetric expression of the phase factor $Q$, namely

$$
\begin{aligned}
& Q= \\
& -\sum_{j=2}^{n} s_{j}\left(\sigma_{j} \theta_{j} h_{j}+\cdots+\sigma_{n} \theta_{n} h_{n}\right) \cdot\left(\theta_{j} h_{j}+\cdots+\theta_{n} h_{n}\right)+H \cdot \underbrace{\sum_{j=2}^{n} s_{j}\left(\sigma_{j} \theta_{j} h_{j}+\cdots+\sigma_{n} \theta_{n} h_{n}\right)}_{=: L_{1}} .
\end{aligned}
$$

This serves as a definition of the function $L_{1}$, whose precise value is irrelevant. The important point lies in the fact $L_{1}$ is linear in the $\left\{h_{k}\right\}_{k=1}^{n}$ 's. Note that in (6.8), the $\left\{h_{k}\right\}_{k \in Z}$ 's are thought as (linear) functions of the $\left(\left\{h_{k}\right\}_{k \notin Z},\left\{H_{p}\right\}_{p=2}^{m}\right)$ 's, according to formula (6.7). We keep on using this convention from now on. We have obtained

$$
\begin{aligned}
& Q=-\sum_{j, \ell=2}^{n}\left[\frac{\sigma_{\ell}+\sigma_{j}}{2}\left(\theta_{\ell} \cdot \theta_{j}\right) S_{\ell \wedge j}\right] h_{j} \cdot h_{\ell}+H \cdot L_{1}, \\
& \text { where } S_{j}=s_{2}+\cdots+s_{j}\left(=t_{1}-t_{j}\right), \quad i \wedge j=\min (i, j) .
\end{aligned}
$$

Second, we may sort out again in (6.9) the dependence of $Q$ on the $\left\{h_{k}\right\}_{k \notin Z}$ 's and on the $\left\{H_{p}\right\}_{p=2}^{m}$ 's, upon writing

$$
\begin{aligned}
& \sum_{j, \ell=2}^{n} \cdots=\sum_{j, \ell \notin Z} \cdots+\sum_{j \notin Z, \ell \in Z} \cdots+\sum_{j \in Z, \ell \notin Z} \cdots+\sum_{j, \ell \in Z} \cdots \\
& \text { and } h_{z_{p}}=\sum_{q \in C_{p}} H_{q}+\sum_{j \in E_{p}} \pi_{p, j} h_{j},
\end{aligned}
$$

see Lemma 1. The second equality holds for any creation momentum $h_{k}$ with $k \in Z$. This procedure provides the identity (note that for any $(j, p)$ such that $j \in E_{p}$, we necessarily have $j \notin Z$ )

$$
Q=-\sum_{j, \ell \notin Z} A_{j, \ell} h_{j} \cdot h_{\ell}+H \cdot L_{1}+H \cdot L_{2}+q(H, H)
$$

where $L_{2}$ is linear in the $\left\{h_{k}\right\}_{k=1}^{n}, q(H, H)$ is quadratic in $H$, the exact value of $L_{2}$ and $q$ is irrelevant, and the important factor is

$$
\begin{aligned}
A_{\ell, j} & =\frac{\sigma_{\ell}+\sigma_{j}}{2}\left(\theta_{\ell} \cdot \theta_{j}\right) S_{\ell \wedge j}+\sum_{p \mid \ell \in E_{p}} \pi_{p, \ell} \frac{\sigma_{z_{p}}+\sigma_{j}}{2}\left(\theta_{z_{p}} \cdot \theta_{j}\right) S_{z_{p} \wedge j} \\
& +\sum_{q \mid j \in E_{q}} \pi_{q, j} \frac{\sigma_{z_{q}}+\sigma_{\ell}}{2}\left(\theta_{z_{q}} \cdot \theta_{\ell}\right) S_{z_{q} \wedge \ell}+\sum_{\substack{p, q \mid \ell \in E_{p}, j \in E_{q}}} \pi_{p, \ell} \pi_{q, j} \frac{\sigma_{z_{p}}+\sigma_{z_{q}}}{2}\left(\theta_{z_{p}} \cdot \theta_{z_{q}}\right) S_{z_{p} \wedge z_{q}} .
\end{aligned}
$$


It may be useful to write down the diagonal term's explicit value:

$$
\begin{aligned}
A_{\ell, \ell} & =2 \sigma_{\ell} S_{\ell} \\
& +\sum_{p \mid \ell \in E_{p}} \pi_{p, \ell}\left(\sigma_{z_{p}}+\sigma_{\ell}\right)\left(\theta_{z_{p}} \cdot \theta_{\ell}\right) S_{z_{p}}+\sum_{p, q \mid \ell \in E_{p} \cap E_{q}} \pi_{p, \ell} \pi_{q, \ell} \frac{\sigma_{z_{p}}+\sigma_{z_{q}}}{2}\left(\theta_{z_{p}} \cdot \theta_{z_{q}}\right) S_{z_{p} \wedge z_{q}} .
\end{aligned}
$$

Third, plugging (6.11) into (6.3), and rescaling

$$
H_{p} \mapsto \varepsilon \widetilde{H}_{p}, \quad(p=2, \ldots, m),
$$

gives the formula (here $\left.\widetilde{H}=\left(\varepsilon H_{1}, \varepsilon H_{2}, \ldots, \varepsilon H_{m}\right)\right)$

$$
\begin{aligned}
& \left|\widehat{\mathcal{T}}\left(t, \xi_{1}, \eta_{1}\right)\right| \leq \varepsilon^{-n / 2} \sum_{\sigma_{1}, \ldots, \sigma_{n}= \pm 1} \int_{0}^{t} d t_{1} \int_{0}^{t_{1}} d t_{2} \int_{0}^{t_{2}} d t_{3} \cdots \int_{0}^{t_{n-1}} d t_{n} \\
& \mid \int \prod_{j \notin Z} d h_{j} \int \prod_{p=2}^{m} d \widetilde{H_{p}} \prod_{j \notin Z} \widehat{\phi}\left(h_{j}\right) \prod_{j \in Z} \widehat{\phi}\left(h_{j}\right) \widehat{f_{m}^{0}}\left(\xi+\widetilde{H} ; \eta+t \xi+\frac{T}{\varepsilon}\right) \\
& \quad \exp \left(\frac{i}{2}\left[L+\widetilde{H} \cdot L_{1}+\widetilde{H} \cdot L_{2}+\varepsilon q(\widetilde{H}, \widetilde{H})\right]\right) \exp \left(-\frac{i}{2 \varepsilon} \sum_{j, \ell \notin Z} A_{j, \ell} h_{j} \cdot h_{\ell}\right) \mid .
\end{aligned}
$$

Finally we remove the $T / \varepsilon$ dependence of $\widehat{f_{m}^{0}}$, upon introducing the Fourier inverse transform $\widetilde{f_{m}^{0}}$ with respect to the $\eta$ variables. Since $T$ is linear in $\left\{\widetilde{H}_{j}\right\}_{j=2}^{m}$ as well as in the $\left\{h_{j}\right\}_{j \notin Z}$ 's, this operation simply changes the linear part of the phase. The final structure of the term under consideration is

$$
\begin{aligned}
& \left|\widehat{\mathcal{T}}\left(t, \xi_{1}, \eta_{1}\right)\right| \leq \varepsilon^{-n / 2} \sum_{\sigma_{1}, \ldots, \sigma_{n}= \pm 1} \int_{0}^{t} d t_{1} \int_{0}^{t_{1}} d t_{2} \int_{0}^{t_{2}} d t_{3} \cdots \int_{0}^{t_{n-1}} d t_{n} \\
& \mid \int \prod_{j \notin Z} d h_{j} \int \prod_{p=2}^{m} d \widetilde{H}_{p} \int \prod_{j=1}^{m} d v_{j} \prod_{j \notin Z} \widehat{\phi}\left(h_{j}\right) \prod_{j \in Z} \widehat{\phi}\left(h_{j}\right) \\
& \widetilde{f_{m}^{0}}\left(\xi+\widetilde{H} ; V_{m}\right) \exp \left(i \alpha+i \sum_{j \notin Z} \beta_{j} h_{j}\right) \exp \left(-\frac{i}{2 \varepsilon} \sum_{j, \ell \notin Z} A_{j, \ell} h_{j} \cdot h_{\ell}\right) \mid,
\end{aligned}
$$

where $V_{m}=\left(v_{1}, \ldots v_{m}\right)$, while $\alpha$ and the $\left\{\beta_{j}\right\}_{j \notin Z}$ 's are suitable (real-valued) functions of $\varepsilon, V_{m},\left(\widetilde{H_{2}}, \ldots \widetilde{H_{m}}\right)$, and the times $s_{1}, \ldots, s_{n}$. Their explicit value is irrelevant in the sequel. The only important point is that $\alpha$ and the $\left\{\beta_{j}\right\}_{j \notin Z}$ 's do not depend on the $\left\{h_{j}\right\}_{j \notin Z}$ 's.

Unfortunately we still cannot apply directly the stationary phase theorem because the integration over the $\left\{h_{j}\right\}_{j \notin Z}$ 's would produce a factor

$$
\frac{\varepsilon^{\frac{d}{2}(n-m+1)}}{|\operatorname{det} A|^{\frac{d}{2}}}
$$

where $A=\left[A_{i j}\right]_{i, j \notin Z}$. The factor $\varepsilon^{\frac{d}{2}(n-m+1)}$ is more than we need to kill $\varepsilon^{-\frac{n}{2}}$. Indeed we know from [BCEP1] that the right graphs are $O(1)$ and estimate (6.17) would give us the contradictory information that such contribution should behave as $O\left(\varepsilon^{\frac{d}{4} n}\right)$. The point is, $|\operatorname{det} A|^{-\frac{d}{2}}$ is not integrable in general with respect to the variables $s_{j}$, as follows by analyzing simple examples. Thus we have to proceed more carefully. The main and somehow surprising point is that we can avoid to detect explicitly the singular manifold of $|\operatorname{det} A|^{-1}$, by using a suitable interpolation technique (see the next step). For the moment we outline the basic features of the matrix A. 
Lemma 4. Setting $q=n-m+1$. Then $A$ is a $q \times q$ symmetric matrix such that

i) if $i<j, A_{i j}$ depends, at most, on the time differences $s_{2}, \ldots, s_{i}$.

ii) $A_{i i}=2 \sigma_{i} s_{i}+G_{i}\left(s_{2}, \ldots s_{i-1}\right)$ where $G_{i}$ is a suitable function of the $i-2$ time differences $s_{2}, \ldots, s_{i-1}$.

Proof of Lemma 4: By direct inspection using that $i \in E_{p}$ implies $i>z_{p}$.

Third step: applying the stationary phase theorem

For later convenience we renumber the entries of the matrix $A$ whose indices are $j_{1}, \ldots j_{q}$, i.e. the indices of the recollision times, by setting

$$
D_{i \ell}=\frac{1}{\varepsilon} A_{j_{i} j_{\ell}}
$$

We also denote

$$
k_{i}=h_{j_{i}}
$$

We finally rescale the recollision time differences by setting

$$
\mu_{i}=\frac{s_{j_{i}}}{\varepsilon}
$$

With these new notations we may estimate

$$
\begin{gathered}
\left|\widehat{\mathcal{T}}\left(t, \xi_{1}, \eta_{1}\right)\right| \leq \varepsilon^{q-n / 2} \sum_{\sigma_{1}, \ldots \sigma_{n}= \pm 1} \int_{0}^{t} d t_{z_{2}} \int_{0}^{t_{z_{2}}} d t_{z_{3}} \cdots \int_{0}^{t_{z_{m}-1}} d t_{z_{m}} \\
\int d V_{m} \int \prod_{p=2}^{m} d \widetilde{H}_{p} \int_{\mathbb{R}^{q}} \prod_{j=1}^{q} d \mu_{j} \mid \int \prod_{j=1}^{q} d k_{j} \prod_{j=1}^{q} \widehat{\phi}\left(k_{j}\right) \prod_{j \in Z} \widehat{\phi}\left(h_{j}\right) \\
\widetilde{f_{m}^{0}}\left(\xi+\widetilde{H} ; V_{m}\right) \exp \left(i \sum_{j=1}^{q} B_{j} \cdot k_{j}\right) \exp \left(-\frac{i}{2} \sum_{i, j=1}^{q} D_{i j} k_{i} \cdot k_{j}\right) \mid,
\end{gathered}
$$

where $B_{\ell}=\beta_{j_{\ell}}$ and the $\left\{h_{j}\right\}_{j \in Z}$ 's have to be thought as functions of $\left\{\widetilde{H}_{j}\right\}_{j=2}^{m}$ and $\left\{k_{j}\right\}_{j=1}^{q}$, according to (6.7). Note that $q-n / 2=n / 2-(m-1)>0$. Therefore it is enough to show that the integrals in the right hand side of (6.19) are uniformly bounded. In that direction, we establish the following

Proposition 3. Let $F: \mathbb{R}^{d q} \rightarrow \mathbb{C}$ be a smooth function (in the sense that the norm defined in (6.21) below is finite), $B \in \mathbb{R}^{d q}, D$ be the $q \times q$ matrix defined in (6.18). Then the following estimate holds true

$$
\begin{aligned}
& \int_{\mathbb{R}^{q}} \prod_{j=1}^{q} d \mu_{j}\left|\int_{\mathbb{R}^{d q}} \prod_{j=1}^{q} d k_{j} F\left(k_{1}, \ldots k_{q}\right) \exp \left(i \sum_{j=1}^{q} B_{j} \cdot k_{j}\right) \exp \left(-\frac{i}{2} \sum_{i, j=1}^{q} D_{i j} k_{i} \cdot k_{j}\right)\right| \\
& \leq C \tilde{\mathcal{N}}(F)
\end{aligned}
$$

where

$$
\tilde{\mathcal{N}}(F)=\max _{I \subset I_{q}} \int d K_{I_{q} \backslash I} d X_{I}\left|\mathcal{F}_{I} F\left(K_{I_{q} \backslash I}, X_{I}\right)\right| .
$$

Here $I_{q}=\{1, \ldots q\}$ and $\mathcal{F}_{I} F\left(K_{I_{q} \backslash I}, X_{I}\right)$ denotes the Fourier transform of $F$ with respect to the variables indexed by $I$. Moreover $C$ is a positive constant independent of $V_{m}, \widetilde{H}$ and the creation times.

\section{Proof of Proposition 3:}

We begin the proof by establishing the following lemma which will be useful in the sequel. 
Lemma 5. Let $\left\{a_{i}\right\}_{i=1}^{k}$ and $\left\{x_{i}\right\}_{i=1}^{k}$ be two collections of real numbers. Let $p>1$. Then, we have the estimate

$$
\int_{\mathbb{R}} \frac{d z}{\left(\sum_{i=1}^{k}\left|a_{i}\right|+\sum_{i=1}^{k}\left|a_{i} z+x_{i}\right|\right)^{p}} \leq \frac{1}{\left(\sum_{i=1}^{k}\left|a_{i}\right|\right)^{p}} \int_{\mathbb{R}} \frac{d z}{(1+|z|)^{p}}
$$

Remark 10. While it is trivial to estimate the left-hand-side of (6.22) by const $/\left|a_{i}\right|$ for any given $i$, the above Lemma allows to keep the improved estimate by const $/\left(\sum_{i}\left|a_{i}\right|\right)^{p}$.

\section{Proof of Lemma 5:}

It is enough to consider the case when $\sum_{i}\left|a_{i}\right|>0$.

We first estimate

$$
\int_{\mathbb{R}} \frac{d z}{\left(\sum_{i=1}^{k}\left|a_{i}\right|+\sum_{i=1}^{k}\left|a_{i} z+x_{i}\right|\right)^{p}} \leq \int_{\mathbb{R}} \frac{d z}{\left(\sum^{*}\left|a_{i}\right|+\sum^{*}\left|a_{i} z+x_{i}\right|\right)^{p}},
$$

where the symbol $\sum^{*}$ designates $\sum_{i: a_{i} \neq 0}$. Next, by convexity, we may write

$$
\sum^{*}\left|a_{i} z+x_{i}\right|=\sum^{*}\left|a_{i}\right|\left|z+\frac{x_{i}}{\left|a_{i}\right|}\right| \geq\left(\sum^{*}\left|a_{i}\right|\right)\left(|z+\underbrace{\sum^{*} \frac{x_{i}}{\left(\sum^{*}\left|a_{i}\right|\right)}}_{=: \bar{x}}|\right) .
$$

The change of variables $z+\bar{x} \mapsto z$ in the above integral finishes the proof.

By the Parseval formula and the explicit form of the Fourier transform of a complex Gaussian, we have

$$
\begin{aligned}
\mathcal{I}:= & \int_{\mathbb{R}^{d q}} \prod_{j=1}^{q} d k_{j} F\left(k_{1}, \ldots k_{q}\right) e^{i \sum_{j=1}^{q} B_{j} \cdot k_{j}} e^{-\frac{i}{2} \sum_{i, j=1}^{q} D_{i j} k_{i} \cdot k_{j}} \\
& =\frac{(2 \pi)^{\frac{d q}{2}} e^{-i \pi \frac{d}{4} \operatorname{sign}(D)}}{|\operatorname{det} D|^{\frac{d}{2}}} \int_{\mathbb{R}^{d q}} d \xi e^{\frac{i}{2} \sum_{i j} D_{i j}^{-1} \xi_{i} \cdot \xi_{j}} \widehat{F}(\xi-B)
\end{aligned}
$$

where $\operatorname{sign}(D)=n^{+}-n^{-}, n^{ \pm}$being the number of positive and negative eigenvalues of $D$ respectively. Therefore

$$
|\mathcal{I}| \leq \frac{C^{q}}{|\operatorname{det} D|^{\frac{d}{2}}}\|\widehat{F}\|_{L_{1}}
$$

More generally, for any subset $I \subset I_{q}$ of indices (including the case $I=\emptyset$, for which $\operatorname{det} D_{I}=1$ and $\mathcal{F}_{\mathcal{I}}=$ identity), we also have

$$
|\mathcal{I}| \leq \frac{C^{q}}{\left|\operatorname{det} D_{I}\right|^{\frac{d}{2}}}\left\|\mathcal{F}_{I} F\right\|_{L_{1}}
$$

where $D_{I}=\left[D_{i j}\right]_{i, j \in I}$. As a consequence

$$
\sum_{I \subset I_{q}}\left|\operatorname{det} D_{I}\right||\mathcal{I}|^{\frac{2}{d}} \leq \tilde{\mathcal{N}}(F)^{\frac{2}{d}} C^{q}
$$

from which we finally deduce

$$
|\mathcal{I}| \leq \frac{C^{q} \widetilde{\mathcal{N}}(F)}{\left(\sum_{I \subset I_{q}}\left|\operatorname{det} D_{I}\right|\right)^{\frac{d}{2}}}
$$


Let now $I \subset I_{q}$ be such that $q \in I$. We evaluate the determinant of $D_{I}$ by using Lemma 4 . Developing along the last row, we compute:

$$
\operatorname{det} D_{I}=2 \sigma_{j_{q}} \mu_{q} \operatorname{det} D_{I \backslash\{q\}}+g_{I \backslash\{q\}, q},
$$

where $g_{I \backslash\{q\}, q}$ is a function of the $\mu_{j}$ 's with $j<q$ only (the value of this function depends on the choice of the index $q$, as well as on the choice of the subset $I \backslash\{q\})$. Therefore

$$
\begin{aligned}
\sum_{I \subset I_{q}}\left|\operatorname{det} D_{I}\right| & =\sum_{I: q \notin I}\left|\operatorname{det} D_{I}\right|+\sum_{I: q \in I}\left|\operatorname{det} D_{I}\right| \\
& =\sum_{I: q \notin I}\left|\operatorname{det} D_{I}\right|+\sum_{I: q \in I}\left|2 \sigma_{j_{q}} \mu_{q} \operatorname{det} D_{I \backslash\{q\}}+g_{I \backslash\{q\}, q}\right| \\
& =\sum_{I: q \notin I}\left|\operatorname{det} D_{I}\right|+\sum_{I: q \notin I}\left|2 \sigma_{j_{q}} \mu_{q} \operatorname{det} D_{I}+g_{I, q}\right|,
\end{aligned}
$$

where the last equality comes from changing the summation index in the second sum. Now, from (6.26) and (6.28), we recover

$$
\begin{aligned}
& \int \prod d \mu_{j}|\mathcal{I}| \leq C^{q} \tilde{\mathcal{N}}(F) \int \prod_{j<q} d \mu_{j} \int d \mu_{q} \frac{1}{\left(\sum_{I \subset I_{q}}\left|\operatorname{det} D_{I}\right|\right)^{\frac{d}{2}}} \\
& =C^{q} \tilde{\mathcal{N}}(F) \int \prod_{j<q} d \mu_{j} \int d \mu_{q} \frac{1}{\left(\sum_{I: q \notin I}\left|\operatorname{det} D_{I}\right|+\sum_{I: q \notin I}\left|2 \sigma_{j_{q}} \mu_{q} \operatorname{det} D_{I}+g_{I, q}\right|\right)^{\frac{d}{2}}} .
\end{aligned}
$$

Applying Lemma 5 therefore yields

$$
\int \prod d \mu_{j}|\mathcal{I}| \leq C^{q} \tilde{\mathcal{N}}(F) \int \prod_{j<q} d \mu_{j} \frac{1}{\left(\sum_{I: q \notin I}\left|\operatorname{det} D_{I}\right|\right)^{\frac{d}{2}}}
$$

There remains to iterate the procedure and to integrate successively over $\mu_{q-1}, \mu_{q-2}, \ldots$ up to $\mu_{1}$. The iteration ends by using det $D_{\emptyset}=1$. This finishes the proof of Proposition 3 .

\section{Fourth step: concluding the proof of Proposition 2}

Inserting estimate (6.20) in (6.19), with

$$
F\left(k_{1}, \ldots k_{q}\right)=\prod_{i=1}^{q} \widehat{\phi}\left(k_{i}\right) \prod_{p=2}^{m} \widehat{\phi}\left(\varepsilon \sum_{r \in C_{p}} \widetilde{H}_{r}+\sum_{\ell: j_{\ell} \in E_{p}} \pi_{p, j_{\ell}} k_{\ell}\right)
$$

we readily see that the Proposition is proved once $\widetilde{\mathcal{N}}(F)$ is conveniently estimated.

We first observe, setting $G^{A}(K)=G(K) e^{i A \cdot K}$, where $A \in \mathbb{R}^{d q}$ and $K=\left(k_{1}, \ldots, k_{q}\right)$, that

$$
\tilde{\mathcal{N}}\left(G^{A}\right)=\tilde{\mathcal{N}}(G) .
$$

This follows by an elementary computation. Now, thanks to (6.30), we have

$$
F(K)=\int_{\mathbb{R}^{d(m-1)}} d Y \prod_{i=2}^{m} \phi\left(y_{i}\right) e^{-i(a+b K) \cdot Y} \prod_{i=1}^{q} \widehat{\phi}\left(k_{i}\right),
$$

where $a \in \mathbb{R}^{d(m-1)}$ and $b \in \mathbb{R}^{d q \times d(m-1)}$ are suitable functions of $\varepsilon$ and the $\left\{\widetilde{H}_{i}\right\}_{i=2}^{m}$ 's. Using (6.31), we thus obtain

$$
\tilde{\mathcal{N}}(F) \leq \int_{\mathbb{R}^{d(m-1)}} d Y \prod_{i=2}^{m}\left|\phi\left(y_{i}\right)\right| \tilde{\mathcal{N}}\left(e^{-i b K \cdot Y} \prod_{i=1}^{q} \widehat{\phi}\left(k_{i}\right)\right) \leq\|\phi\|_{L_{1}}^{m-1} c^{q} \widetilde{\mathcal{N}}(\widehat{\phi})^{q} \leq c^{n} \tilde{\mathcal{N}}(\widehat{\phi})^{n} .
$$


In conclusion, we have established

$$
\left|\widehat{\mathcal{T}}\left(t, \xi_{1}, \eta_{1}\right)\right| \leq \varepsilon^{\frac{n}{2}-m+1} \frac{t^{m-1}}{(m-1) !} c^{n}\left(\|\phi\|_{L_{1}}+\|\widehat{\phi}\|_{L_{1}}\right)^{n}\left\|\widetilde{f}_{0}\right\|_{L_{1}}^{m-1}\left\|f_{0}\right\|_{L_{1}} .
$$

This concludes the proof of Proposition 2, and ends this paragraph.

\section{Right non-ordered graphs}

The previous analysis readily establishes the estimate $\widehat{\mathcal{T}}\left(t, \xi_{1}, \eta_{1}\right) \leq O\left(\varepsilon^{n / 2-(m-1)}\right)$ in full generality. In the particular case when $m-1=n / 2$, this reduces to $\widehat{\mathcal{T}}\left(t, \xi_{1}, \eta_{1}\right) \leq O(1)$. An additional argument is needed to prove that right non-ordered graphs, or wrong graphs with $m-1>n / 2$, are actually vanishing as $\varepsilon \rightarrow 0$.

We start by considering right non-ordered graphs, reminding that they involve as many creation as recollision events (i.e. $\left.\frac{n}{2}=m-1\right)$. Besides, for any creation event, they involve exactly one associated recollision between the same particles (i.e. between the father and the son). However the time ordering of the recollision is not the right one: at least one recollision does not happen immediately after the associated creation.

It turns out that a slight improvement of the arguments developed in the previous paragraph allows to exploit this wrong time ordering so as to prove $\widehat{\mathcal{T}}\left(t, \xi_{1}, \eta_{1}\right) \leq O\left(\varepsilon^{\gamma}\right)$, for some $\gamma>0$. Lemma 2 is crucial in that respect.

Before coming to the proof, we first state the main result of this paragraph.

Proposition 4. Let $\mathcal{T}(t)$ be a right non-ordered graph such that $m-1=n / 2$. Then, under the smoothness assumptions of our main Theorem, there is a constant $C=C\left(d, m, n, \phi, f_{0}, t\right)$, which depends on all the mentioned arguments but not on $\varepsilon$, such that

$$
\left\|\widehat{\mathcal{T}}\left(t, \xi_{1} ; \eta_{1}\right)\right\|_{L^{\infty}\left(\mathbb{R}^{d} \times \mathbb{R}^{d}\right)} \leq C \varepsilon^{\gamma}, \quad \text { with } 0<\gamma<\frac{d-2}{2} .
$$

Remark 11. The dependence of $C$ upon the various parameters in Proposition 4 is of the form

$$
C \leq c(d)^{n}\left(\|\widehat{\phi}\|_{L_{1}}+\|\phi\|_{L_{1}}\right)^{n} \mathcal{N}_{\alpha}\left(f_{0}\right)^{m} \frac{t^{m-2}}{(m-2) !}
$$

where $c(d)$ only depends on the dimension.

Remark 12. In this part of the proof we do not require the assumption $\widehat{\phi}(0)=0$.

\section{Proof of Proposition 4}

The idea is to come back to the computation of the diagonal elements of the quadratic phase $Q$, see (6.13).

For any given particle $p$, denote by $i(p)$ the index of the (unique) recollision between particle $p$ and $p$ 's father. This notation is well-defined since the graph is assumed right non-ordered. With these notations, the index $i(p)$ belongs to the set $E_{p}$ (and to no other set $E_{q}$ with $q \neq p$ ). Besides, we have $\theta_{z_{p}}=\theta_{i(p)}$, and $\pi_{p, i(p)}=-1$. As a consequence, the diagonal coefficient $A_{i(p), i(p)}$ has the simple value (see (6.13))

$$
\begin{aligned}
A_{i(p), i(p)} & =2 \sigma_{i(p)} S_{i(p)}-2\left(\sigma_{z_{p}}+\sigma_{i(p)}\right) S_{z_{p}}+2 \sigma_{z_{p}} S_{z_{p}} \\
& =2 \sigma_{i(p)}\left(S_{i(p)}-S_{z_{p}}\right) 2 \sigma_{i(p)}\left[s_{z_{p}+1}+s_{z_{p}+2}+\cdots+s_{i(p)}\right] .
\end{aligned}
$$

The important point is, not only the recollision time $s_{i(p)}$ appears explicitly in (7.1), but also the intermediate times $s_{z_{p}+1}, s_{z_{p}+2}$, etc.

Now, since the graph is assumed right non-ordered, we may apply Lemma 2: there is a particle $q$ and a particle $p$, with $q<p$, such that

$$
z_{p}<z_{q}<i(p) \text {. }
$$


For this particular choice of $p$ (and $q$ ), we recover

$$
A_{i(p), i(p)}=2 \sigma_{i(p)}\left[s_{z_{p}+1}+s_{z_{p}+2}+\cdots+s_{z_{q}}+\cdots+s_{i(p)}\right]=2 \sigma_{i(p)}\left[s_{z_{q}}+\alpha\right],
$$

where $\alpha$ is a sum of positive terms. We may now improve Proposition 3. By using (6.26), summing only over $I=\{i(p)\}$ and $I=\emptyset$ we obtain, for $\mathcal{I}$ given in (6.23), the following bound

$$
|\mathcal{I}| \leq \frac{c \mathcal{N}_{0}(F)}{\left(1+2\left(\frac{s_{z_{q}}}{\varepsilon}+\alpha\right)\right)^{\frac{d}{2}}} \leq \frac{c \mathcal{N}_{0}(F)}{\left(1+2 \frac{s_{z_{q}}}{\varepsilon}\right)^{\frac{d}{2}}}
$$

Note that $s_{z_{q}}$ is a creation time, and hence it is not rescaled. Obviously $F$ in (7.4) is given by (6.30). Interpolating equation (7.4) and equation (6.26) we arrive at

$$
|\mathcal{I}| \leq \frac{c \mathcal{N}_{0}(F)}{\left(1+2 \frac{s_{z_{q}}}{\varepsilon}\right)^{\frac{d}{2} \beta}\left(\sum_{I}\left|\operatorname{det} D_{I}\right|\right)^{\frac{d}{2}(1-\beta)}},
$$

where $0<\beta<\frac{d-2}{d}$. Inserting (7.5) in (6.19) we proceed as in section 6 replacing $\frac{d}{2}$ by $\frac{d}{2}(1-\beta)>1$. The final result follows by

$$
\int_{0}^{t} \frac{d s}{\left(1+2 \frac{s}{\varepsilon}\right)^{\frac{d}{2} \beta}} \leq c \varepsilon^{\frac{d}{2} \beta} t^{1-\frac{d}{2} \beta} .
$$

Remark 13. A remark is in order. In [BCEP1] we learnt that a quantum collision in a weak coupling regime, as expressed by an operator $C_{i, j}^{\varepsilon}$, is not "completed": it needs an operator $T_{i, j}^{\varepsilon}$, expressing a proper recollision, and occurring approximatively at the same time, in order to give a contribution $O(1)$. Therefore one may conceive that a right non-ordered graph forces the creation times occurring between pairs of creation-recollision times, to give a relevant contribution on a set of small measure only. Unfortunately we cannot fully exploit this feature since we are not able to explicitly characterize the singular manifold of $(\operatorname{det} D)^{-1}$.

\section{Graphs for which $m-1>n / 2$.}

The previous two paragraphs use a stationary phase approach to balance the factor $\varepsilon^{-d(m-1)-n / 2}$ in the definition of $\widehat{\mathcal{T}}$, and to eventually prove $\widehat{\mathcal{T}} \rightarrow 0$ as $\varepsilon \rightarrow 0$. The main point is (up to the slight refinement of paragraph 7 ) the quadratic phase $Q$ allows to gain one factor $\varepsilon$ per recollision time. This approach seems difficult to adapt in the case $m-1>n / 2$ : it would require a very fine control of various coefficients in the quadratic phase $Q$, combined with suitable cancellation effects due to the signs $\sigma_{j}$, in order to recover additional factors $\varepsilon$ from creation times as well.

For that reason, in this paragraph and the next one we renounce to control the phase and we follow a quite different route: we exploit the assumption $\widehat{\phi}(0)=0$ to balance some divergence arising from small exchanged momenta.

The main result of this paragraph is the

Proposition 5. Let $\mathcal{T}(t)$ be a graph such that $m-1>n / 2$. Then, under the smoothness assumptions of our main theorem, and provided the potential $\phi$ satisfies $\widehat{\phi}(0)=0$, there is a constant $C=C\left(d, m, n, \phi, f_{0}, t\right)$, which depends on all the mentioned arguments but not on $\varepsilon$, such that

$$
\left\|\widehat{\mathcal{T}}\left(t, \xi_{1} ; \eta_{1}\right)\right\|_{L^{\infty}\left(\mathbb{R}^{d} \times \mathbb{R}^{d}\right)} \leq C \varepsilon^{m-1-n / 2} \underset{\varepsilon \rightarrow 0}{\longrightarrow} 0
$$


Remark 14. The dependence of $C$ upon the various parameters in Proposition 5 is of the form

$$
C \leq c(d)^{n} \mathcal{N}_{\alpha}(\phi)^{n} \mathcal{N}_{\alpha}\left(f_{0}\right)^{m} \frac{t^{n-(m-1)}}{(n-(m-1)) !}
$$

where $c(d)$ only depends on the dimension.

\section{Proof of Proposition 5}

To begin with, we first bound $\widehat{\mathcal{T}}$, as given through formula (3.19), by

$$
\begin{array}{r}
\left|\widehat{\mathcal{T}}\left(\xi_{1}, \eta_{1}, t\right)\right| \leq 2^{n}\left\|\widehat{f}_{0}\right\|_{\infty} \varepsilon^{-d(m-1)-\frac{n}{2}} \int_{0}^{t} d t_{1} \int_{0}^{t_{1}} d t_{2} \cdots \int_{0}^{t_{n-1}} d t_{n} \\
\int_{\mathbb{R}^{n d}} d h_{1} d h_{2} \cdots d h_{n} \prod_{j=1}^{n}\left|\widehat{\phi}\left(h_{j}\right)\right| \prod_{k=2}^{m}\left|\widehat{f}_{0}\left(\frac{e_{k} \cdot H}{\varepsilon}, \frac{e_{k} \cdot T}{\varepsilon}\right)\right| .
\end{array}
$$

Note the phase factor $\exp \left(i S_{\varepsilon} / \varepsilon\right)$ has been estimated by one here: stationary phase considerations are completely put apart from now on.

Now, the point is, the variables $e_{k} \cdot T$ involve a particular triangular structure. Namely, for all $k=2, \ldots, m$, we have

$$
e_{k} \cdot T=t_{z_{k}} h_{z_{k}}+A_{k}
$$

where $A_{k}$ is a linear combination of $t_{j}$ and $h_{j}$ with $j>z_{k}$. In particular, variable $t_{z_{2}}$ is only involved in $e_{2} \cdot T$, variable $t_{z_{3}}$ is only involved in $e_{2} \cdot T$ and $e_{3} \cdot T$, etc. For that reason, we may successively perform the integrations in $t_{z_{2}}, \ldots, t_{z_{m}}$ in (8.1), as follows. The integration in $t_{z_{2}}$ produces the factor

$$
\int d t_{z_{2}}\left|\widehat{f_{0}}\left(\frac{H_{2}}{\varepsilon}, \frac{t_{z_{2}} h_{z_{2}}+A_{2}}{\varepsilon}\right)\right| \leq c \mathcal{N}_{\alpha}\left(f_{0}\right) \frac{\varepsilon}{\left|h_{z_{2}}\right|}\left(1+\left|\frac{H_{2}}{\varepsilon}\right|^{2}\right)^{-\frac{\alpha}{2}} .
$$

Note also that the integration in $t_{z_{2}}$ leaves all other factors $\widehat{f}_{0}\left(e_{3} \cdot H / \varepsilon, e_{3} \cdot T / \varepsilon\right), \ldots, \widehat{f}_{0}\left(e_{m} \cdot H / \varepsilon, e_{m} \cdot T / \varepsilon\right)$ unchanged in (8.1). Next, the integration in $t_{z_{3}}$ again produces a factor

$$
\int d t_{z_{3}}\left|\widehat{f_{0}}\left(\frac{H_{3}}{\varepsilon}, \frac{t_{z_{3}} h_{z_{3}}+A_{3}}{\varepsilon}\right)\right| \leq c \mathcal{N}_{\alpha}\left(f_{0}\right) \frac{\varepsilon}{\left|h_{z_{3}}\right|}\left(1+\left|\frac{H_{3}}{\varepsilon}\right|^{2}\right)^{-\frac{\alpha}{2}}
$$

while leaving all other factors $\widehat{f}_{0}\left(e_{4} \cdot H / \varepsilon, e_{4} \cdot T / \varepsilon\right), \ldots \widehat{f}_{0}\left(e_{m} \cdot H / \varepsilon, e_{m} \cdot T / \varepsilon\right)$ unchanged in (8.1). Performing this procedure until $t_{z_{m}}$, and last integrating over all other time variables $\left\{t_{j}\right\}_{j \notin Z}$ as well, we eventually obtain

$$
\begin{aligned}
\left|\widehat{\mathcal{T}}\left(\xi_{1}, \eta_{1}, t\right)\right| \leq c^{n} \mathcal{N}_{\alpha}\left(f_{0}\right)^{m} \varepsilon^{-d(m-1)-\frac{n}{2}+(m-1)} \frac{t^{n-(m-1)}}{(n-(m-1)) !} \\
\int_{\mathbb{R}^{n d}} d h_{1} d h_{2} \cdots d h_{n} \prod_{j \in Z} \frac{\left|\widehat{\phi}\left(h_{j}\right)\right|}{\left|h_{j}\right|} \prod_{j \notin Z}\left|\widehat{\phi}\left(h_{j}\right)\right| \prod_{k=2}^{m}\left(1+\left|\frac{H_{k}}{\varepsilon}\right|^{2}\right)^{-\frac{\alpha}{2}} .
\end{aligned}
$$

Recall variables $H_{k}=H \cdot e_{k}$ are thought as functions of $\left(h_{1}, \ldots, h_{n}\right)$ as before.

There remains to observe the assumption $\widehat{\phi}(0)=0$, in conjunction with the smoothness of $\widehat{\phi}$, readily gives

$$
\frac{|\widehat{\phi}(h)|}{|h|} \leq\|D \widehat{\phi}\|_{L^{\infty}}
$$


From this it follows

$$
\begin{array}{r}
\left|\widehat{\mathcal{T}}\left(\xi_{1}, \eta_{1}, t\right)\right| \leq c^{n}\|D \widehat{\phi}\|_{L^{\infty}}^{m-1} \mathcal{N}_{\alpha}\left(f_{0}\right)^{m} \varepsilon^{-d(m-1)-\frac{n}{2}+(m-1)} \frac{t^{n-(m-1)}}{(n-(m-1)) !} \\
\int_{\mathbb{R}^{n d}} d h_{1} d h_{2} \cdots d h_{n} \prod_{j \notin Z}\left|\widehat{\phi}\left(h_{j}\right)\right| \prod_{k=2}^{m}\left(1+\left|\frac{H_{k}}{\varepsilon}\right|^{2}\right)^{-\frac{\alpha}{2}} .
\end{array}
$$

Last, we rescale the creation momenta as in (6.6) and (6.14). We arrive at

$$
\begin{gathered}
\left|\widehat{\mathcal{T}}\left(\xi_{1}, \eta_{1}, t\right)\right| \leq c^{n}\|D \widehat{\phi}\|_{L^{\infty}}^{m-1} \mathcal{N}_{\alpha}\left(f_{0}\right)^{m} \varepsilon^{-\frac{n}{2}+(m-1)} \frac{t^{n-(m-1)}}{(n-(m-1)) !} \\
\prod_{k=2}^{m} \int_{\mathbb{R}^{d}} d \widetilde{H_{k}}\left(1+\left|\widetilde{H}_{k}\right|^{2}\right)^{-\frac{\alpha}{2}} \prod_{j \notin Z} \int_{\mathbb{R}^{d}} d h_{j}\left|\widehat{\phi}\left(h_{j}\right)\right| .
\end{gathered}
$$

Hence, reminding that $\alpha>d$,

$$
\left|\widehat{\mathcal{T}}\left(\xi_{1}, \eta_{1}, t\right)\right| \leq c(d)^{n} \mathcal{N}_{\alpha}\left(f_{0}\right)^{m} \mathcal{N}_{\alpha}(\phi)^{n} \frac{t^{n-(m-1)}}{(n-(m-1)) !} \varepsilon^{-\frac{n}{2}+(m-1)} .
$$

This finishes the proof of Proposition 5.

\section{$9 \quad$ Wrong graphs with $m-1=n / 2$}

The previous paragraph establishes the estimate $\widehat{\mathcal{T}}\left(t, \xi_{1}, \eta_{1}\right) \leq O\left(\varepsilon^{m-1-n / 2}\right)$ in full generality, provided the potential satisfies $\widehat{\phi}(0)=0$. In the particular case when $m-1=n / 2$, this estimate is useless. An additional argument is needed to prove wrong graphs with $m-1=n / 2$ actually have vanishing contribution as $\varepsilon \rightarrow 0$.

We remind that wrong graphs with $m-1=n / 2$ are characterized by Lemma 3 established in paragraph 5. Now, a slight modification of the argument used in the previous paragraph allows us to exploit this specific feature, and prove $\widehat{\mathcal{T}}\left(t, \xi_{1}, \eta_{1}\right)=O(\varepsilon)$.

The main result of this paragraph is the

Proposition 6. Let $\mathcal{T}(t)$ be a wrong graph such that $m-1=n / 2$. Then, under the smoothness assumptions of our main theorem, and provided the potential $\phi$ satisfies $\widehat{\phi}(0)=0$, there is a constant $C=C\left(d, m, n, \phi, f_{0}, t\right)$, which depends on all the mentioned arguments but not on $\varepsilon$, such that

$$
\left\|\widehat{\mathcal{T}}\left(t, \xi_{1} ; \eta_{1}\right)\right\|_{L^{\infty}\left(\mathbb{R}^{d} \times \mathbb{R}^{d}\right)} \leq C \varepsilon \underset{\varepsilon \rightarrow 0}{\longrightarrow} 0 .
$$

Remark 15. The dependence of $C$ upon the various parameters in Proposition 5 is of the form

$$
C \leq c(d)^{n} \mathcal{N}_{\alpha}(\phi)^{n} \mathcal{N}_{\alpha}\left(f_{0}\right)^{m} \frac{t^{n-(m-1)}}{(n-(m-1)) !}
$$

where $c(d)$ only depends on the dimension.

\section{Proof of Proposition 6}

We simply modify the argument of the previous paragraph by using Lemma 3 .

According to Lemma 3 , there is a particle $p$ such that $\left|E_{p}\right| \geq 2$, and there exists one index $i \in E_{p}$ such that $\pi_{p, i}=-1$. On the other hand, and as before, for any $k=2, \ldots, m$, we have

$$
e_{k} \cdot T=t_{z_{k}} h_{z_{k}}+A_{k}
$$


where $A_{k}$ is a linear combination of $t_{j}$ and $h_{j}$ with $j>z_{k}$. Yet the situation is a bit more precise in the special case $k=p$. For this particular value, we may write indeed

$$
e_{p} \cdot T=t_{z_{p}} h_{z_{p}}+t_{i} h_{i}+\widetilde{A_{p}}
$$

where $\widetilde{A_{p}}$ is a linear combination of $t_{j}$ 's and $h_{j}$ 's, $j>z_{p}, j \neq i$. Notice that both variables $t_{z_{p}}$ and $t_{i}$ only appear in quantities of the form $e_{j} \cdot T$ with $j<p$ : due to the information $\pi_{p, i}=-1$, we know indeed the partner particle in the $i$-th recollision is created before particle $p$.

These informations allow to perform successively the integrations in the variables $t_{z_{2}}, t_{z_{3}}, \ldots, t_{z_{p}}, t_{i}, t_{z_{p+1}}$, ..., $t_{z_{m}}$ in the estimate

$$
\begin{array}{r}
\left|\widehat{\mathcal{T}}\left(\xi_{1}, \eta_{1}, t\right)\right| \leq c^{n}\left\|\widehat{f}_{0}\right\|_{\infty} \varepsilon^{-d(m-1)-\frac{n}{2}} \int_{0}^{t} d t_{1} \int_{0}^{t_{1}} d t_{2} \cdots \int_{0}^{t_{n-1}} d t_{n} \\
\int_{\mathbb{R}^{n d}} d h_{1} d h_{2} \cdots d h_{n} \prod_{j=1}^{n}\left|\widehat{\phi}\left(h_{j}\right)\right| \prod_{k=2}^{m}\left|\widehat{f}_{0}\left(\frac{e_{k} \cdot H}{\varepsilon}, \frac{e_{k} \cdot T}{\varepsilon}\right)\right| .
\end{array}
$$

We already discussed the effect of the integrations over the $t_{z_{k}}$ 's $(k \neq p)$ in the previous paragraph. On the other hand, integration over $t_{z_{p}}$ and $t_{i}$ produces the factor

$$
\begin{gathered}
\int_{\mathbb{R}} d t_{z_{p}} \int_{\mathbb{R}} d t_{i}\left|\widehat{f_{0}}\left(\frac{e_{p} \cdot H}{\varepsilon}, \frac{t_{z_{p}} h_{z_{p}}+t_{i} h_{i}+\widetilde{A_{p}}}{\varepsilon}\right)\right| \\
\leq \mathcal{N}_{\alpha}\left(f_{0}\right) \frac{\varepsilon^{2}}{\operatorname{area}\left(h_{z_{p}}, h_{i}\right)}\left(1+\left|\frac{e_{p} \cdot H}{\varepsilon}\right|^{2}\right)^{-\frac{\alpha}{2}},
\end{gathered}
$$

where area $\left(h_{z_{p}}, h_{i}\right)$ denotes the area of the two-dimensional parallelogram defined by the two vectors $h_{z_{p}}$ and $h_{i}$. Its value is,

$$
\begin{aligned}
\operatorname{area}\left(h_{z_{p}}, h_{i}\right) & =\left|h_{z_{p}}\right|\left|h_{i}-\left[h_{i} \cdot \frac{h_{z_{p}}}{\left|h_{z_{p}}\right|}\right] \frac{h_{z_{p}}}{\left|h_{z_{p}}\right|}\right| \\
& =\left|h_{z_{p}}\right|\left|h_{i}\right|\left|\frac{h_{i}}{\left|h_{i}\right|}-\left[\frac{h_{i}}{\left|h_{i}\right|} \cdot \frac{h_{z_{p}}}{\left|h_{z_{p}}\right|}\right] \frac{h_{z_{p}}}{\left|h_{z_{p}}\right|}\right| .
\end{aligned}
$$

Hence the whole integration procedure over variables $t_{z_{2}}, t_{z_{3}}, \ldots, t_{z_{p}}, t_{i}, t_{z_{p+1}}, \ldots, t_{z_{m}}$ eventually gives the estimate

$$
\begin{gathered}
\left|\widehat{\mathcal{T}}\left(\xi_{1}, \eta_{1}, t\right)\right| \leq c^{n} \mathcal{N}_{\alpha}\left(f_{0}\right)^{m} \varepsilon^{-d(m-1)-\frac{n}{2}+(m-1)+1} \frac{t^{n-(m-1)-1}}{(n-(m-1)-1) !} \\
\int_{\mathbb{R}^{n d}} d h_{1} d h_{2} \cdots d h_{n} \prod_{j \in Z, j \neq z_{p}} \frac{\left|\widehat{\phi}\left(h_{j}\right)\right|}{\left|h_{j}\right|} \prod_{j \notin Z, j \neq i}\left|\widehat{\phi}\left(h_{j}\right)\right| \\
\frac{\left|\widehat{\phi}\left(h_{i}\right)\right|\left|\widehat{\phi}\left(h_{z_{p}}\right)\right|}{\left|\operatorname{area}\left(h_{z_{p}}, h_{i}\right)\right|} \prod_{k=2}^{m}\left(1+\left|\frac{H_{k}}{\varepsilon}\right|^{2}\right)^{-\frac{\alpha}{2}} .
\end{gathered}
$$

The role of indices $i$ and $p$ is clearly put apart here. Here and below, the reader may keep in mind the relation $m-1=n / 2$.

Let us now estimate the right-hand-side of (9.5). To do so, let $\ell$ be the second external recollision in $E_{p}$ (see Lemma 3). Separating the role of $\ell$ as well, and rescaling the creation momenta $H_{k}(k=2, \ldots, m)$ as in (6.6) 
and (6.14), we recover

$$
\begin{array}{r}
\left|\widehat{\mathcal{T}}\left(\xi_{1}, \eta_{1}, t\right)\right| \leq c^{n} \mathcal{N}_{\alpha}\left(f_{0}\right)^{m} \mathcal{N}_{\alpha}(\phi)^{m-2} \varepsilon \frac{t^{m-2}}{(m-2) !} \int \prod_{j \notin Z} d h_{j} \int \prod_{k \in Z} d \widetilde{H_{k}} \\
\prod_{j \notin Z, j \neq i, \ell}\left|\widehat{\phi}\left(h_{j}\right)\right| \frac{\left|\widehat{\phi}\left(h_{i}\right)\right|\left|\widehat{\phi}\left(h_{z_{p}}\right)\right|\left|\widehat{\phi}\left(h_{\ell}\right)\right|}{\left|\operatorname{area}\left(h_{z_{p}}, h_{i}\right)\right|} \prod_{k=2}^{m}\left(1+\left|\widetilde{H_{k}}\right|^{2}\right)^{\frac{\alpha}{2}} .
\end{array}
$$

Recall here that the variables $\left\{h_{k}\right\}_{k \in Z}$ 's are thought as linear functions of the $\left\{h_{j}\right\}_{j \notin Z}$ 's and the $\left\{\widetilde{H_{k}}\right\}_{k=2}^{m}$ 's. In that respect, we actually know from Lemma 1 that

$$
h_{z_{p}}=\varepsilon \sum_{q \in C_{p}} \widetilde{H_{q}}-h_{i}+\pi_{p, \ell} h_{\ell}+\sum_{j \in E_{p}, j \neq i, \ell} \pi_{p, j} h_{j} .
$$

This suggests the change of variable

$$
h_{\ell} \longrightarrow k=\varepsilon \sum_{q \in C_{p}} \widetilde{H_{q}}-h_{i}+\pi_{p, \ell} h_{\ell}+\sum_{j \in E_{p}, j \neq i, \ell} \pi_{p, j} h_{j} .
$$

in (9.6). Indeed, changing variables in this way we arrive at the estimate

$$
\begin{aligned}
& \left|\widehat{\mathcal{T}}\left(\xi_{1}, \eta_{1}, t\right)\right| \leq c^{n} \mathcal{N}_{\alpha}\left(f_{0}\right)^{m} \mathcal{N}_{\alpha}(\phi)^{m-2+n-(m-1)-2} \varepsilon \frac{t^{m-2}}{(m-2) !} \\
& \int_{\mathbb{R}^{2 d}} d h_{i} d k \frac{\left|\widehat{\phi}\left(h_{i}\right)\right||\widehat{\phi}(k)|\left|\widehat{\phi}\left(h_{\ell}\right)\right|}{\left|\operatorname{area}\left(k, h_{i}\right)\right|}
\end{aligned}
$$

Here $h_{\ell}$ is a function of the $\widetilde{H}_{j}$ 's with $j \in C_{p}, k$, and some $h_{j}$ 's with $j \in E_{p}$. There remains to observe the estimate (here we denote $\left.\widehat{k}:=k /|k|, \widehat{h_{i}}:=h_{i} /\left|h_{i}\right|\right)$,

$$
\begin{array}{rl}
\int_{\mathbb{R}^{2 d}} & d h_{i} d k \frac{\left|\widehat{\phi}\left(h_{i}\right)\right||\widehat{\phi}(k)|\left|\widehat{\phi}\left(h_{\ell}\right)\right|}{\left|\operatorname{area}\left(k, h_{i}\right)\right|} \\
& =\int_{\mathbb{R}^{2 d}} d h_{i} d k \frac{\left|\widehat{\phi}\left(h_{i}\right)\right||\widehat{\phi}(k)|\left|\widehat{\phi}\left(h_{\ell}\right)\right|}{|k|\left|h_{i}\right|\left[\widehat{h_{i}}-\left(\widehat{h_{i}} \cdot \widehat{k}\right) \widehat{k}\right]} \\
& \leq \mathcal{N}_{d}(\phi)^{3} \int_{\mathbb{S}^{d-1} \times \mathbb{S}^{d-1}} d \sigma d \sigma^{\prime} \frac{1}{\left|\sigma-\left(\sigma \cdot \sigma^{\prime}\right) \sigma^{\prime}\right|} \\
& \leq c(d) \mathcal{N}_{d}(\phi)^{3},
\end{array}
$$

where $c(d)$ is some universal constant, depending on the dimension $d$ only. Here we used the fact that $d \geq 3$. Eventually we have proved

$$
\left|\widehat{\mathcal{T}}\left(\xi_{1}, \eta_{1}, t\right)\right| \leq c(d)^{n} \mathcal{N}_{\alpha}\left(f_{0}\right)^{m} \mathcal{N}_{\alpha}(\phi)^{n} \varepsilon \frac{t^{m-2}}{(m-2) !} .
$$

This finishes the proof of Proposition 6.

\section{References}

[AM] N.W. Ashcroft, N.D. Mermin, Solid stats physics, Saunders, Philadelphia (1976).

[Ba] R. Balescu, Equilibrium and Nonequilibrium Statistical Mechanics, John Wiley \& Sons, New-York (1975). 
[BCEP1] D. Benedetto, F. Castella, R. Esposito, M. Pulvirenti, Some Considerations on the derivation of the nonlinear Quantum Boltzmann Equation, J. Stat. Phys., Vol. 116, No. 114, pp. 381-410 (2004).

[BCEP2] D. Benedetto, F. Castella, R. Esposito, M. Pulvirenti, On The Weak-Coupling Limit for Bosons and Fermions, Math. Mod. Meth. Appl. Sci., Vol. 15, No. 12, pp. 1811-1843 (2005).

[BCEP3] D. Benedetto, F. Castella, R. Esposito, M. Pulvirenti, Some Considerations on the derivation of the nonlinear Quantum Boltzmann Equation II: the low-density regime, J. Stat. Phys., Vol. Online First, DOI: 10.1007/s10955-005-9010-x (2006)

[Bo] A. Bohm, Quantum Mechanics, Texts and monographs in Physics, Springer-Verlag (1979).

[C] F. Castella, From the von Neumann equation to the Quantum Boltzmann equation II: identifying the Born series, J. Stat. Phys., Vol. 106, No. 5/6, pp. 1197-1220 (2002).

[CC] S. Chapman, T. G. Cowling, The Mathematical Theory of Non-uniform Gases, Cambridge Univ. Press, Cambridge, England (1970).

[CIP] C. Cercignani, R. Illner, M. Pulvirenti, The mathematical theory of dilute gases, Applied Mathematical Sciences, Vol. 106, Springer-Verlag, New York (1994).

[Ch] S.L. Chuang, Physics of optoelectronic devices, Wiley series in pure and applied optics, New-York (1995).

[CTDL] C. Cohen-Tannoudji, B. Diu, F. Laloë, Mécanique Quantique, I et II, Enseignement des Sciences, Vol. 16, Hermann (1973).

[DGL] D. Dürr, S. Goldstain, J.L. Lebowitz Asymptotic motion of a classical particle in a random potential in two dimensions: Landau model, Comm. Math. Phys., Vol. 113, No. 2, pp. 209-230 (1987).

[EE] D. Eng, L. Erdös, The Linear Boltzmann Equation as the Low Density Limit of a random Schrödinger equation, Rev. Math. Phys., Vol. 17, No. 6, pp. 669-743 (2005).

[EY1] L. Erdös, H.T. Yau, Linear Boltzmann Equation as Scaling Limit of Quantum Lorentz Gas Advances in differential equations and mathematical physics (Atlanta, GA, 1997), Contemp. Math., 217, Amer. Math. Soc., Providence, RI, pp. 137-155 (1998).

[EY2] L. Erdös, H.T. Yau, Linear Boltzmann Equation as the Weak Coupling Limit of a Random Schrödinger Equation, Comm. Pure Appl. Math., Vol. 53, No. 6, pp. 667-735 (2000).

[ESY] L. Erdös, M. Salmhofer, H.-T. Yau, On the quantum Boltzmann equation, J. Stat. Phys., Vol. 116, No. 1-4, pp. 367-380 (2004).

[H] M.N. Hugenholtz, Derivation of the Boltzmann equation for a Fermi gas, J. Stat. Phys., Vol. 32, pp. 231-254 (1983).

[HL] N.T. Ho, L.J. Landau, Fermi gas in a lattice in the van Hove limit, J. Stat. Phys., Vol 87, pp. 821-845 (1997).

[IP] R. Illner, M. Pulvirenti, Global Validity of the Boltzmann equation for a two-dimensional rare gas in the vacuum, Comm. Math. Phys. Vol. 105 189-203 (1986), Erratum and improved result, Comm. Math. Phys., Vol. 121, pp. 143-146 (1989).

[L] O. Lanford III, Time evolution of large classical systems, Lecture Notes in Physics, Vol. 38, pp. 1-111, E.J. Moser ed., Springer-Verlag (1975).

[KP] H. Kesten, G.C. Papanicolaou, A limit theorem for stochastic acceleration. Comm. Math. Phys., Vol. 78, No. 1, 19-63 (1980/81) 
[LP] P.L.Lions, T.Paul, Sur les mesures de Wigner, Revista Matematica ibero americana, Vol. 9, No. 3, pp. 553-618 (1993).

[MRS] P.A. Markowich, C.A. Ringhofer, C. Schmeiser, Semiconductor equations., Springer-Verlag, Vienna (1990).

[RS] M. Reed, B. Simon, Methods of modern mathematical physics III. Scattering theory, Academic Press, New York-London (1979). NON CITATO

[RV] E. Rosencher, B. Vinter, Optoelectronique, Dunod (2002).

[Sp] H. Spohn, Derivation of the transport equation for electrons moving through random impurities, J. Stat. Phys., Vol. 17, No. 6, pp. 385-412 (1977).

[U] K. Uchiyama, On the Boltzmann-Grad limit for the Broadwell model of the Boltzmann equation, Jour. Stat. Phys., Vol. 52, No. 1/2, pp. 331-355 (1988).

[UU] E.A. Uehling, G.E. Uhlenbeck, Transport phenomena in Einstein-Bose and Fermi-Dirac gases. I, Phys. Rev., Vol. 43, pp. 552-561 (1933).

[W] E.P. Wigner, On the quantum correction for thermodynamic equilibrium, Phys. Rev., Vol. 40, pp. 749-759 (1932). 